\title{
Die Entstehung von Vertrauen im Kontext von Unsicherheit und Informationsasymmetrie
}

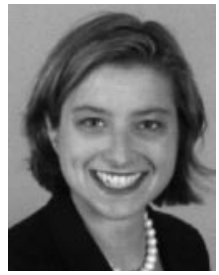

Isabell M. Welpe

\section{Überblick}

- Der Kontext junger Unternehmen ist durch Risiko, Unsicherheit und Informationsasymmetrie gekennzeichnet. Da Verträge in einem solchen Umfeld unvollständig bleiben müssen, ist ein hohes Maß an Vertrauen besonders wichtig für die Entstehung und Aufrechterhaltung inner- und zwischenbetrieblicher Kooperationen.

- Ein zentraler Gegenstand der Kooperation für die wirtschaftlich erfolgreiche Entwicklung junger Unternehmen ist die Zusammenarbeit mit ihren Eigenkapital-Gebern. Die bisherige Forschung schreibt dabei Vertrauen zwischen Unternehmen und ihren Investoren eine Schlüsselrolle für die Entstehung, Aufrechterhaltung und die Qualität der Zusammenarbeit zu. Dennoch gibt es bisher kaum empirische Studien zur Vertrauensbildung im Unternehmensgründungskontext und zur Entstehung von Vertrauen von Unternehmern in ihre Kooperationspartner.

- Das vorrangige Ziel dieser Arbeit ist es daher zu untersuchen, wie Vertrauen der Unternehmer in ihre Investoren entsteht. Dabei differenziert diese Arbeit zwei unterschiedliche Dimensionen von Vertrauen: Zum einen das Vertrauen in den Goodwill (Betreibt der Verhandlungspartner beidseitige Nutzenmaximierung?) und zum anderen das Vertrauen in die Kompetenz (Ist der Verhandlungspartner kompetent und fähig, seine Versprechen zu erfüllen und das zu tun, was er sagt?).

- Die im Rahmen dieser Studie betrachteten Forschungsfragen lauten: Wie entsteht Vertrauen aus Sicht von Unternehmern? Gibt es Unterschiede in der Entstehung von Goodwill- und Kompetenz-Vertrauen? Wie wirken sich erste Interaktionen zwischen den Akteuren auf ihre spätere Zusammenarbeit aus? Die Hypothesenprüfung erfolgt an einer Stichprobe von 272 Venture-Capitalfinanzierten Unternehmen aus dem deutschsprachigen europäischen Raum.

- Die Ergebnisse bestätigen, dass Goodwill- und Kompetenz-Vertrauen zwei eigenständige Dimensionen von Vertrauen sind, welche unterschiedliche Zusammenhangsmuster mit ihren Prädiktoren aufweisen. Die beobachteten Unterschiede in der Beziehung zwischen den beiden Formen des Vertrauens und ihrer Einflussgrößen betonen die Notwendigkeit, zukünftig nicht nur das Ausmaß, sondern auch die Art des Vertrauens in theoretischen und empirischen Arbeiten zu berücksichtigen.

Keywords Trust · information asymmetry $\cdot$ entrepreneurship $\cdot$ goodwill trust $\cdot$ competence trust . pre-contractual $\cdot$ fairness $\cdot$ cooperation

Eingegangen: 7. Mai 2007

JEL: A13, C12, L14

PD Dr. Isabell M. Welpe $(\bowtie)$

Senior Research Fellow, Max Planck Institute of Economics, Entrepreneurship, Growth and Public Policy

Group, Jena,welpe@econ.mpg.de

Institut für Information, Organisation und Management, Ludwig-Maximilians-Universität, München 


\section{A. Einführung}

Die Frage, wie Vertrauen entsteht, wird sowohl in der allgemeinen betriebswirtschaftlichen (Korsgaard, Pitariu \& Jeong, im Erscheinen; Schoorman, Mayer \& Davis, 2007), (neuro)ökonomischen (Damasio, 2005; Kosfeld, Heinrichs, Zak, Fischbacher \& Fehr, 2005) und psychologischen (Ferrin, Dirks \& Shah, 2006) Forschung als auch in der Entrepreneurship-Forschung ${ }^{1}$ (Welter \& Smallbone, 2006; Mesquita, 2007; Morse, Fowler \& Lawrence, 2007) mit zunehmender Bedeutung diskutiert.

Der Kontext der vorliegenden Studie ${ }^{2}$ ist in besonderem Maß geeignet, um die Entstehung von Vertrauen unter Bedingungen hoher Unsicherheit und des Fehlens von vertraglichen Absicherungsmöglichkeiten zu untersuchen. Die bisherige Forschung hat gezeigt, dass die Umgebung junger Unternehmen durch hohes Risiko (Grichnik \& Immerthal, 2005; Zahra, Yavuz \& Ucbasaran, 2006), Informationsasymmetrie (Amit, Glosten \& Muller, 1990), Unsicherheit (Aldrich \& Auster, 1986) und die Gefahr opportunistischen Verhaltens (Cable \& Shane, 1997) gekennzeichnet ist. Daraus folgt, dass nicht alle möglicherweise auftretenden Entwicklungen im Voraus vertraglich geregelt werden können und Verträge in der Regel unvollständig bleiben müssen (Williamson, 1985; Manigart, Korsgaard, Folger, Sapienza \& Baeyens, 2001). In einem derart charakterisierten Umfeld kommt Vertrauen für das Zustandekommen und Aufrechterhalten inter-organisationaler Beziehungen eine Schlüsselrolle zu (Larson, 1992; Mayer, Davis \& Schoorman, 1995).

Eine zentrale zwischenbetriebliche Beziehung für junge Wachstumsunternehmen ist die Zusammenarbeit mit Eigenkapital-Investoren ${ }^{3}$ (Cable \& Shane, 1997) wie VentureCapital-Gebern (Kollmann \& Kuckertz, 2006). Frühere Forschungsarbeiten zeigen, dass für den Erfolg und die Entwicklung von jungen Unternehmen die Qualität dieser Zusammenarbeit und Kooperation wichtiger sein kann als das investierte Kapital selbst (Timmons \& Bygrave, 1986; Sapienza \& Korsgaard, 1996; Schefczyk, 1999). Eine Reihe theoretisch-konzeptioneller Studien betont ausdrücklich die Rolle von Vertrauen für die Qualität der Zusammenarbeit zwischen Eigenkapital-Investoren und Unternehmern (bspw. De Clercq \& Sapienza, 2001; Shepherd \& Zacharakis, 2001). Gleichwohl gibt es bisher kaum empirische Arbeiten, welche die Entstehung von Vertrauen im Gründungskontext allgemein oder in Beziehungen junger Unternehmen mit ihren Geschäftspartnern oder Investoren thematisieren. Welter und Smallbone (2006) bemerken, dass Vertrauen im Bereich Entrepreneurship - anders als bspw. in der Organisationsforschung - erst in den letzten Jahren größere Aufmerksamkeit geschenkt wurde. Sie betonen weiter, dass die Frage, wie Vertrauen entsteht, nach wie vor offen und wenig erforscht ist: „An open question is how to best build trust. Trust building is an under researched topic" (Welter \& Smallbone, 2006: 471).

Die wenigen empirischen Arbeiten bezüglich der Antezedentien von Vertrauen in diesem Kontext wurden zudem entweder aus Sicht der Eigenkapital-Geber (EKG) (Sapienza \& Korsgaard, 1996; Harrison, Dibben \& Mason, 1997; Amaeshi \& Scarbrough, 2006; Kollmann \& Kuckertz, 2006) oder in Beziehungen zu Fremdkapitalgebern (vgl. Saparito, 2000; Saparito, Chen \& Sapienza, 2004; Howorth \& Moro, 2006; Ngyen, Le \& Freeman, 2006) analysiert. Wir wissen daher nur wenig über die Entstehung des Vertrauens von Unternehmern in ihre Eigenkapital-Investoren, z.B. wie das unternehmerische Vertrauen in ihre Investoren beschaffen ist und welche Faktoren es beeinflussen können (Manigart 
et al., 2001). Hinzu kommt, dass frühere Studien zu Vertrauen im Unternehmensgründungskontext Vertrauen zumeist als eindimensionales und undifferenziertes Konzept betrachtet haben, was jedoch nicht dem aktuellen Forschungsstand zu Vertrauen entspricht (Mayer et al., 1995; Neergard \& Ullhøi, 2006). Allgemein anerkannt sind zwei unterschiedliche Dimensionen von Vertrauen. Danach resultiert Vertrauen sowohl aus dem Urteil über die Fähigkeit oder Kompetenz eines anderen („Kompetenz-Vertrauen“) als auch aus der Einschätzung der Wahrscheinlichkeit, dass ein Akteur keine Vereinbarungen bricht und eine einseitige Nutzenmaximierung betreibt (,Goodwill4-Vertrauen“) (Sako, 1992; 1995; Jones \& George, 1998). ${ }^{5}$

Vor diesem Hintergrund ist es Ziel dieser Arbeit, die Entstehung von Goodwill- und Kompetenz-Vertrauen im von Unsicherheit und Risiko geprägten Kontext junger Unternehmen zu untersuchen. Die dieser Arbeit zugrunde liegenden Forschungsfragen sind daher: Wie entsteht Vertrauen aus Sicht von Unternehmensgründern? Gibt es Unterschiede in der Entstehung von Goodwill- und Kompetenz-Vertrauen? Wie wirken sich die ersten Interaktionen zwischen den Kooperationspartnern auf das Goodwill-und Kompetenz-Vertrauen in ihrer späteren Zusammenarbeit aus?

Zur Beantwortung der Forschungsfragen untersucht die vorliegende Studie die Bedeutung der ersten, vor-vertraglichen Interaktionen zwischen EKG und Unternehmen für die Entstehung von Vertrauen in der nach-vertraglichen Phase. Dabei unterscheidet sie die vor-vertraglichen Interaktionen nach inhaltlichen Aspekten und Prozessaspekten. Um die Beschaffenheit des unternehmerischen Vertrauens besser verstehen zu können, unterscheidet diese Studie außerdem zwischen Goodwill- und Kompetenz-Vertrauen und untersucht deren Prädiktoren unter Heranziehung von erhobenen Primärdaten von 272 VentureCapital-finanzierten Unternehmen aus dem deutschsprachigen Europa.

Die vorliegende Arbeit baut zum Teil auf früheren Studien auf, welche das Konzept des Kompetenz-Vertrauens beschrieben haben (z.B. Sako, 1992; Mayer et al., 1995), und erweitert diese, indem sie zwischen Kompetenz- und Goodwill-Vertrauen nicht nur konzeptionell unterscheidet, sondern diesen Unterschied auch empirisch analysiert, was frühere Studien oftmals unberücksichtigt gelassen haben (Barney \& Hansen, 1994; Jones \& George, 1998).

Die Ergebnisse dieser Studie tragen auf mehrfache Weise zum aktuellen Forschungsstand bei: Sie bestätigen empirisch, dass Goodwill- und Kompetenz-Vertrauen unterschiedliche und eigenständige Konstrukte darstellen. Sie zeigen, dass Goodwill- und Kompetenz-Vertrauen unterschiedlichen Einflussfaktoren unterliegen. Ein besseres Verständnis der Determinanten der Vertrauensbildung präzisiert sowohl die allgemeine Vertrauensliteratur als auch die Literatur über inter-organisationale Beziehungen, indem sie Einblicke in die Schaffung von Vertrauen in multilateralen Allianzen gewährt. Weiterhin tragen die Ergebnisse über die Beziehung zwischen Investoren und jungen Unternehmen zum Forschungsfeld der Entrepreneurial Finance bei. Zudem sind die Ergebnisse für ein besseres Verständnis kontext-spezifischer Modelle von Vertrauen relevant, wie bspw. von Schoorman et al. (2007) gefordert, da sie zum Verständnis von Vertrauen in kooperativen Beziehungen mit hoher Unsicherheit und in denjenigen Situationen beitragen, in denen ein Austritt aus der Beziehung kostspielig und schwierig ist. Außerdem leistet diese Studie einen Beitrag zur Schließung der durch Landström (1990) hervorgehobenen Forschungslücke in Bezug auf die frühen Interaktionen zwischen Venture-Capital-Geber und Wachs- 
tumsunternehmen, indem sie explizit Einflussfaktoren auf Vertrauen aus der vor-vertraglichen Phase der Zusammenarbeit betrachtet.

Diese Arbeit wird im Folgenden in vier Abschnitte untergliedert. Zuerst werden in Abschnitt B relevante Theorien und Ergebnisse früherer Studien über die Determinanten von Vertrauen diskutiert. In Abschnitt $\mathrm{C}$ werden ein Bezugsrahmen sowie Hypothesen über die Vorläufer von Goodwill- und Kompetenz-Vertrauen in Eigenkapital-Investitionen aus der Sichtweise der Unternehmer aufgestellt. Abschnitt D präsentiert die Methodologie, Abschnitt E die empirischen Ergebnisse, welche in Abschnitt F diskutiert werden.

\section{B. Goodwill- und Kompetenz-Vertrauen im Risiko- und Unsicherheitskontext junger Unternehmen}

"We need each other. We're in the same lifeboat; once we've cut the mooring and written the check and are rowing out to sea with each other with the guy (sic), we can either pull in the same direction or we can hit each other over the head with the oars"

Zitat eines Venture-Capital-Gebers in Sapienza (1989)

Die bisherige Forschung belegt, dass kooperative Zusammenarbeit zwischen EKG und Unternehmer eine notwendige (aber nicht hinreichende) Bedingung für die erfolgreiche Entwicklung junger Unternehmen ist (Cable \& Shane, 1997). Eine spieltheoretische Betrachtung der Beziehung zwischen EKG und Unternehmen verdeutlicht, dass beide Akteure die Möglichkeit haben, sowohl opportunistisch zu handeln als auch durch eine gegenseitige Kooperation ihren Nutzen zu maximieren (Cable \& Shane, 1997). Trotz starker Kooperationsanreize zeigen jedoch sowohl Forschungsarbeiten als auch Beispiele aus der Praxis, dass bestmögliche Kooperation zwischen diesen beiden Akteuren alles andere als üblich ist (Sahlman, 1990; Sapienza, 1992; Shepherd \& Zacharakis, 2001).

Cable \& Shane (1997) heben in ihrem theoretischen Modell die Bedeutung von gegenseitigem Vertrauen für eine erfolgreiche Kooperation zwischen EKG und Unternehmen hervor, indem sie deren Beziehung durch das Gefangenendilemma modellieren. Frühere Untersuchungen des Gefangenendilemmas untermauern zudem die Bedeutung von Vertrauen in der Entstehung und Aufrechterhaltung jeglicher Formen erfolgreicher Kooperation (bspw. Albach, 1980; Güth, Ockenfels \& Wendel, 1992), da der theoretische Rahmen des Gefangenendilemmas die Überwindung derjenigen Anreize einbezieht, welche beide Akteure dazu (ver)führen, die eigenen Interessen zu Lasten des Nutzens einer bilateralen Kooperation zu verfolgen (Axelrod \& Dijon, 1988; Güth. et al., 1992). Ein großer Anreiz für unkooperatives Verhalten in der Zusammenarbeit zwischen EKG und Unternehmern ist neben den Opportunitätskosten der Kooperation die große Informationsasymmetrie zwischen beiden Akteuren (Reißig-Thust, Brettel \& Witt, 2004). So haben bspw. Unternehmer bessere Informationen über den Zustand des jungen Unternehmens, seine Technologie und seine zukünftigen Entwicklung, während EKG bspw. mehr Informationen über zukünftige Finanzierungsmöglichkeiten haben (Cable \& Shane, 1997).

Ein besseres Verständnis davon, wie Vertrauen in der Entwicklung bilateraler Kooperation zwischen EKG und den Unternehmern entsteht, ist daher eine dringende For- 
schungsfrage. Bisher wurde Vertrauen jedoch im Kontext junger Unternehmen hauptsächlich aus der Sichtweise des EKG untersucht (Amaeshi \& Scarbrough, 2006), und dabei insbesondere der Nutzen von vertraglichen Kontrollmechanismen für den EKG (Barney, Busenitz, Fiet \& Moesel, 1989; Chan, Siegel \& Thakor, 1990; Steier \& Greenwood, 1995; Hellmann, 1998; Kirilenko, 2001; Hommel, Farag, Witt \& Wright, 2004).

Die vorliegende Arbeit argumentiert jedoch, dass vertragliche „Kontrollmechanismen“ aufgrund einer Reihe von Gründen nicht ausreichend effektiv sind, um das notwendige $\mathrm{Ma}$ an Kooperation zwischen EKG und Unternehmern zu schaffen: Erstens ist der Kontext junger Unternehmen sehr riskant und unsicher (Aldrich \& Auster, 1986; Stuart \& Abetti, 1987; Zahra et al., 2006), und daher kann keiner der Partner vollends kontrollieren und überwachen, ob der andere Partner seinen Teil des Vertrags erfüllt (Cable \& Shane, 1997; Hellmann, 1998; Demougin \& Fabel, 2007; Hellmann, 2007). Zweitens existieren nur wenige Kontrollmechanismen und vertragliche Vereinbarungen für die Unternehmensgründer zur Überwachung der EKG (Hellmann, 1998; Reißig-Thust et al., 2004; Breuer, Antonczyk \& Mark, 2007). Daraus folgt, dass Vertrauen der Unternehmer in den EKG eine notwendige und kaum ersetzbare Bedingung für die Entstehung von Kooperation zwischen dem EKG und den Unternehmern ist.

Dabei ist es plausibel anzunehmen, dass Unternehmer in unterschiedliche Aspekte ihres EKG Vertrauen entwickeln können (Neergard \& Ullhøi, 2006). In der jüngeren Vertrauensforschung gibt es einen generellen Trend (z.B. Borys \& Jemison, 1989; Ring \& van de Ven, 1992; Sako, 1992; Ganesan, 1994; Doney \& Cannon, 1997; Das \& Teng, 2001; Ba \& Pavlou, 2002; Pavlou, 2002; Green, 2003), eine Unterscheidung zwischen zwei Dimensionen von Vertrauen in (a) Kompetenz-Vertrauen und in (b) Goodwill-Vertrauen vorzunehmen. Obwohl in bisherigen wissenschaftlichen Arbeiten wiederholt darauf hingewiesen wurde, dass es sinnvoll wäre, in empirischen Untersuchungen zwischen unterschiedlichen Arten und Dimensionen von Vertrauen zu unterscheiden, ist der Frage nach der Entwicklung und den Funktionen von Goodwill- und Kompetenz-Vertrauen im Kontext junger Unternehmen bisher wenig theoretische und empirische Aufmerksamkeit geschenkt worden (Blomqvist, 2002).

Da Goodwill-Vertrauen und Kompetenz-Vertrauen konzeptionell und inhaltlich unterschiedliche Konstrukte sind, ist es nahe liegend anzunehmen, dass sie auch in unterschiedlicher Weise geschaffen werden und dass sie möglicherweise unterschiedliche Auswirkungen haben. Bisher ist jedoch nicht bekannt, wodurch Kompetenz-Vertrauen geschaffen wird ${ }^{6}$ und wie Kompetenz- und Goodwill-Vertrauen in Beziehung zueinander stehen. Für ein besseres Verständnis der Entstehungs- und Wirkungsweise von Vertrauen ist aber eine Unterscheidung zwischen Kompetenz- und Goodwill-Vertrauen auch im spezifischen Kontext junger Unternehmen, welcher von Risiko, Unsicherheit und Informationsasymmetrien gekennzeichnet ist, von Bedeutung. Es ist weiterhin plausibel anzunehmen, dass Goodwillund Kompetenz-Vertrauen auf unterschiedliche Weise verletzt werden können, unterschiedliche Maßnahmen einen Verlust von Kompetenz- und Goodwill-Vertrauen kompensieren können und nicht vorhandenes Goodwill- und Kompetenz-Vertrauen unterschiedliche Konsequenzen für die Entwicklung und den Erfolg unternehmerischen Handelns haben.

Abbildung 1 differenziert zwischen einem hohen und niedrigen Grad an Goodwillund Kompetenz-Vertrauen und zeigt beispielhaft mögliche Konsequenzen einer Verletzung dieser beiden Formen des Vertrauens in der Kooperation zwischen EKG und 


\begin{tabular}{|c|c|c|c|}
\hline & \multicolumn{2}{|c|}{ Goodwill-Vertrauen } \\
\hline & & Hoch & Niedrig \\
\hline 흘 & Hoch & $\begin{array}{c}\text { Hohe } \\
\text { Kooperationsgewinne } \\
\text { Optimaler Transfer } \\
\text { von nicht-finanziellen Leistungen }\end{array}$ & $\begin{array}{l}\text { Kooperationsverluste } \\
\text { Ausgleich möglich durch ... } \\
\text {... Erhöhung Anreizkompatibilität } \\
\text {... vertragliche Anreize } \\
\text {... Anteil am Unternehmen }\end{array}$ \\
\hline 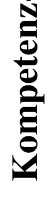 & $\begin{array}{c}\text { Nied- } \\
\text { rig }\end{array}$ & $\begin{array}{l}\text { Kooperationsverluste } \\
\text { Ausgleich möglich durch ... } \\
\text {... Erhöhung Fachkompetenz } \\
\text {... weitere Investoren } \\
\text {... Hinzulernen }\end{array}$ & $\begin{array}{c}\text { Hohe } \\
\text { Kooperationsverluste } \\
\text { Kaum Ausgleich möglich ... } \\
\text { Kaum Transfer } \\
\text { von nicht-finanziellen Leistungen }\end{array}$ \\
\hline
\end{tabular}

Abb. 1. Die möglichen Folgen unterschiedlich hohen Goodwill- und Kompetenz-Vertrauens in der Kooperation zwischen Eigenkapital-Geber und Unternehmen aus Sicht der Unternehmer.

Unternehmer auf. Es ist in der bisherigen Forschung zur Zusammenarbeit von VentureCapital-Firmen und ihren Portfolio-Unternehmen gut dokumentiert, dass der eigentlich wertsteigernde Effekt der Zusammenarbeit mit Venture-Capital-Gebern über den Transfer finanzieller Leistungen von Venture-Capital-Gebern an Unternehmen hinausgeht (Brettel, Thust \& Witt, 2001) und vor allem durch die nicht-finanziellen Leistungen der Venture-Capital-Geber wie Beratung und Unterstützung bedingt ist (MacMillan, Kulow \& Khoylian, 1989; Schefczyk \& Gerpott, 1998; Gerpott \& Schefczyk, 2001a; Welpe, 2004; Meier, Hiddemann \& Brettel, 2006; Stubner, 2007). Ein Transfer nicht-materieller Ressourcen setzt jedoch eine funktionierende Kooperation zwischen EKG und Unternehmen voraus (Shepherd \& Zacharakis, 2001; Welpe, 2004).

Abbildung 1 zeigt auch, dass eine Verletzung von Goodwill- und Kompetenz-Vertrauen zu unterschiedlichen Kompensationsmechanismen führen kann. Bei einem Mangel an Vertrauen in die Kompetenz eines Kooperationspartners reagiert man möglicherweise eher mit der Bereitstellung von technischer oder fachlicher Unterstützung oder der Hinzuziehung weiterer Investoren mit Fachkenntnis. Bei einem Mangel an Vertrauen in den Goodwill eines Kooperationspartners bekundet man eventuell eher Unmut (,voice“) (Hirschman, 1970) oder verbessert die vertraglichen Anreize für den Investor oder erhöht den Anteil des Investors am Unternehmen, um eine Interessensangleichung zu schaffen (Milgrom \& Roberts, 1992). Ein optimaler Transfer der nicht-finanziellen Ressourcen in der EKG-Unternehmer-Beziehung ist nur bei gleichzeitig hohem Kompetenz- und Goodwill-Vertrauen möglich. Bei einem Fehlen von sowohl Goodwill- als auch KompetenzVertrauen ist kaum ein Transfer wertsteigernder nicht-finanzieller Ressourcen möglich, und der EKG wird so zum reinen Geldgeber.

In bisherigen Arbeiten zu Vertrauen stand vor allem das Goodwill-Vertrauen im Zentrum der Untersuchung, und Wissenschaftler haben hauptsächlich seine Entstehung und 
Auswirkungen betrachtet (Clark \& Mills, 1979; Clark \& Waddel, 1985; Clark, Mills \& Powell, 1986; Pennings \& Woiceshyn, 1987; Holmes \& Rempel, 1989, Green, 2003; Bunduchi, 2005). Der aktuelle Konsens in der Forschung zu Vertrauen ist, dass fehlendes Goodwill-Vertrauen einer Beziehung mehr schadet als fehlendes Kompetenz-Vertrauen, da Goodwill-Vertrauen allgemein als die bedeutendere Dimension des Vertrauens angesehen wird (Kim, Ferrin, Cooper \& Dirks, 2004).

Die vorliegende Arbeit hinterfragt jedoch, ob in der bisherigen Vertrauensforschung aufgrund der hohen Bewertung, welche Goodwill-Vertrauen erfahren hat, die Rolle von Kompetenz-Vertrauen für die Entwicklung einer Kooperation in einem hoch riskanten und unsicheren Umfeld wie dem Entrepreneurship- und Venture-Capital-Kontext unterschätzt wird. Zum Beispiel könnte fehlendes Vertrauen in die Kompetenz des EKG die Art der Kommunikation des Unternehmers mit dem EKG, die Höhe des Informationsaustausches sowie den Grad, bis zu dem der Unternehmer bereit sein wird, dem Rat des EKG zu folgen, beeinflussen. Letztlich wird dadurch die Qualität der Kooperation und die Wahrscheinlichkeit einer wertschöpfenden Zusammenarbeit zwischen Unternehmern und Investoren beeinflusst (Busenitz, Fiet \& Moesel, 2004). Im Gegensatz zu zahlreichen früheren Studien nimmt diese Arbeit daher an, dass Unternehmer aufgrund der hohen Opportunitätskosten einer Kooperation und der großen Informationsasymmetrie im Kontext der Entwicklung junger Firmen sowohl in den Goodwill als auch in die Kompetenz ihrer EKG vertrauen müssen, um erfolgreiche Kooperationen eingehen und aufrecht erhalten zu können.

Ein weiterer Aspekt der Fragestellungen dieser Studie bezieht sich auf die explizite Einbeziehung der vor-vertraglichen Phase in die empirische Untersuchung. Da frühere Studien die Interaktion zwischen EKG und Unternehmern, die vor der Unterzeichnung des Investitionsvertrages stattgefunden hat, nicht betrachtet haben, ist wenig über den Verhandlungsprozess, die Vertragsfestlegung und Auswirkungen in der so genannten vor-vertraglichen Phase bekannt (Landström, Manigart, Mason \& Sapienza, 1998). Cable \& Shane (1997) haben allerdings festgestellt, dass die vor-vertragliche Phase als ein „Reibungsprozess“ dient, in der durch Identifikation und Lösung von Agency-Problemen Grundlagen für eine langfristige Beziehung zwischen EKG und Unternehmen gelegt werden.

Aus der Vertrauensforschung ist gut bekannt, dass die ersten Eindrücke und Kontakte wichtig sind für die Bildung von Vertrauen (bspw. McEvily, Perrone \& Zaheer, 2003). Daher schlägt diese Studie vor, drei Dimensionen der ersten Interaktion zwischen EKG und Unternehmern in der vor-vertraglichen Phase zu betrachten und die Wirkung dieser Interaktion sowohl auf Goodwill-Vertrauen als auch auf Kompetenz-Vertrauen hin zu untersuchen. Zwei Dimensionen - nämlich „Fairness des Vertrags“ und „Offenheit/Transparenz während der Due Diligence ${ }^{7 *}$ - behandeln dabei die Qualität und den Prozess der Interaktion zwischen EKG und Unternehmen. Die frühere Forschung über verteilte Gerechtigkeit hat die wahrgenommene Fairness im Zustandekommen von Entscheidungen (Cobb, Wooten \& Folger, 1995) bereits untersucht, aber bisher haben lediglich wenige Studien explizit den Einfluss von Fairness in Beziehungen zwischen Unternehmern und EKG untersucht (Landström et al., 1998), und keine Studie hat bisher in diesem Kontext einen Bezug zu Vertrauen hergestellt. Die dritte Dimension - die „Bedarfs- und Bedürfnisanalyse“ - betrachtet den Inhalt der Interaktion zwischen EKG und Unternehmern in der vor-vertraglichen Phase. 
Ziel dieser Studie ist es, zum Verständnis des Wesens und der Funktionen inter-organisationaler Vertrauensbeziehungen beizutragen durch erstens die Unterscheidung zwischen zwei wesentlichen Formen des inter-organisationalen Vertrauens - Kompetenz- und Goodwill-Vertrauen -, zweitens die Untersuchung der Wechselbeziehung dieser beiden Formen des Vertrauens und drittens die Identifizierung von Faktoren, welche die Entwicklung beider Formen von Vertrauen beeinflussen. Diese Studie betrachtet die Bedarfsanalyse, Fairness des Vertrags und Offenheit der Due Diligence als Einflussfaktoren auf die Entwicklung von sowohl Kompetenz- als auch Goodwill-Vertrauen der Unternehmer in ihre EKG.

Zusätzlich zu der Frage, ob die bereits erwähnten Variablen das Vertrauen beeinflussen, interessieren im Rahmen dieser Studie auch Unterschiede in der Größe der Effekte, da aus der gegenwärtigen Forschung nicht bekannt ist, welche Faktoren besonders stark auf Goodwill- und Kompetenz-Vertrauen wirken. Es ist plausibel anzunehmen, dass einige Faktoren aufgrund unterschiedlich wirkender Prozesse stärkeren Einfluss auf Vertrauen haben als andere (Dirks \& Ferrin, 2002).

\section{Entwicklung von Hypothesen: Einflussfaktoren auf Goodwill- und Kompetenz-Vertrauen}

Abbildung 2 stellt den Bezugsrahmen der vorliegenden Untersuchung dar. Die Bedarfsanalyse, Fairness des Vertrags und Offenheit der Due Diligence beziehen sich auf die vor-vertraglichen Interaktionen, während Kompetenz- und Goodwill-Vertrauen sich auf die nach-vertragliche Zusammenarbeit beziehen.

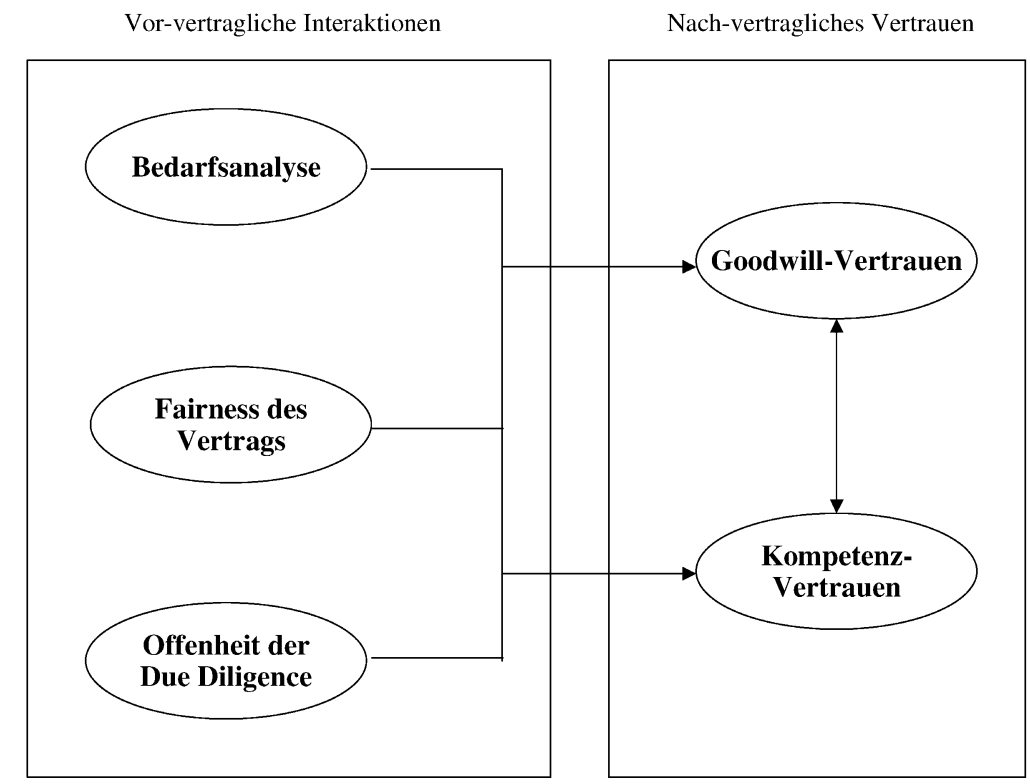

Abb. 2. Konzeptionelles Modell der Studie 
Die erste Hypothese postuliert einen Zusammenhang zwischen beiden Vertrauensarten, die Hypothesen zwei bis vier beziehen sich auf Goodwill-Vertrauen und die Hypothesen fünf bis sieben beziehen sich auf Kompetenz-Vertrauen.

Zusammenhang zwischen Goodwill- und Kompetenz-Vertrauen. Frühere Forschung zu Vertrauen hat gezeigt, dass die verschiedenen Vertrauenskomponenten generell voneinander abhängen (Mayer et al. 1995; McKnight, Cummings \& Chervany, 1998). Analog zu Ganesan (1994), der argumentiert, dass sowohl Goodwill- als auch Kompetenz-Vertrauen Konstrukte sind, welche die Zuversicht in das Verhalten eines Individuums darstellen, postuliert diese Studie, dass eine allgemein positive Korrelation zwischen diesen beiden Konstrukten existiert. Diese Argumentation stimmt auch mit Pavlou (2002) überein, der anhand empirischer Untersuchungen einen starken Zusammenhang zwischen wahrgenommener Kompetenz und Wohlwollen in zwischenbetrieblichen Käufer-Verkäufer-Beziehungen nachweist. Folglich stellt diese Studie die Hypothese auf, dass Goodwill- und Kompetenz-Vertrauen der Unternehmensgründer in ihre Investoren positiv korrelieren. Dementsprechend lautet Hypothese eins:

\section{Hypothese 1: Goodwill-Vertrauen ist positiv mit Kompetenz-Vertrauen assoziiert.}

Bedarfsanalyse. Diese Studie argumentiert, dass die Intensität und das Ausmaß einer Bedarfsanalyse in der vor-vertraglichen Interaktion sowohl zur Schaffung von Goodwill- als auch von Kompetenz-Vertrauen der Unternehmer in den EKG in der weiteren Beziehung beitragen kann, da eine offene Bedarfsanalyse die Möglichkeiten der Unternehmer, die Kompetenzen und Absichten ihrer Investoren wahrzunehmen, erhöht. Eine frühe Offenlegung von Stärken, Schwächen und Bedürfnisse des Unternehmens im Rahmen einer vor-vertraglichen Bedarfsanalyse ermöglicht es, dass sowohl Goodwill- als auch Kompetenz-Vertrauen der Unternehmer in die Investoren entstehen und wahrgenommen werden kann. Erst durch die Kenntnis der Bedürfnisse und Schwächen können die Investoren spezifische Vorschläge für Hilfe und Unterstützung unterbreiten und dabei in den Augen der Unternehmer ihre Kompetenz, ihr Wissen und ihre Fachkenntnisse nachweisen. Dies sollte den Unternehmern ermöglichen, die Kompetenz ihrer Investoren besser zu beurteilen und seine Kompetenz zu erkennen. Eine umfangreiche Kenntnis der Hilfsbedürfnisse, Risiken und Schwächen der Unternehmer und des Unternehmens erleichtert außerdem den EKG die rechtzeitige Informationsbereitstellung und andere Formen der Unterstützung und erhöht so das Vertrauen der Unternehmer in die Kompetenz ihrer Investoren in der Phase der nach-vertraglichen Zusammenarbeit.

Außerdem kann eine vor-vertragliche Bedarfsanalyse auch Goodwill-Vertrauen in der nachfolgenden EKG-Unternehmer-Kooperation verbessern. Die Aufdeckung sensibler Information hängt auch vom Vertrauen in die wohlwollenden und gutartigen Absichten des Kooperationspartners ab (Butler, 1999). Daher besitzen Unternehmen, die ihre Schwächen, Risiken und Bedürfnisse offen legen, meist bereits einen gewissen Grad an Goodwill-Vertrauen in ihre Investoren. Durch die Verhandlung der zukünftigen Kooperation und der Eingestehung ihrer Bedürfnisse bereits zu einem frühen Zeitpunkt der Zusammenarbeit ermöglichen die Unternehmen den EKG, ihren „Goodwill“ in der nachfolgenden Zusammenarbeit unter Beweis zu stellen, indem sie die kommunizierten Schwächen nicht zum Nachteil des Unternehmens ausnutzen. Dadurch, dass die Unternehmen sich verletzbar machen (vgl. Weibel, 2007), ermöglichen sie den EKG zu zei- 
gen, ob sie dem entgegengebrachten Goodwill-Vertrauen der Unternehmen in sie würdig sind.

Hinzu kommt, dass die Durchführung einer eingehenden Beurteilung des Unternehmens viel Zeit und Arbeit vor Unterzeichnung des Investitionsvertrags von Seiten des EKG in Anspruch nimmt. Zeit ist eine der knappsten Ressourcen für Investoren (Smart, 1998). Daher könnten Unternehmen die vom EKG investierte Zeit auch als einen Beweis für den Goodwill des Investors ansehen und in diesen Vertrauen. Hypothese zwei und drei lauten:

Hypothese 2: Je intensiver die Bedarfsanalyse in der vor-vertraglichen Phase ist, desto höher ist das Goodwill-Vertrauen in der nach-vertraglichen Phase.

Hypothese 3: Je intensiver die Bedarfsanalyse in der vor-vertraglichen Phase ist, desto höher ist das Kompetenz-Vertrauen in der nach-vertraglichen Phase.

Fairness des Vertrags. Analog zu dem Bezugssystem von Shepherd und Zacharakis (2001), welches die Bedeutung von Fairness für die Vertrauensbildung in der Kooperation zwischen EKG und Unternehmern betont, argumentiert diese Studie, dass die Fairness der Vertragsverhandlung das Vertrauen des Unternehmers in der nach-vertraglichen Phase auf mehrfache Weise beeinflusst: Erstens erzeugen Verfahren, die strukturell und zwischenbetrieblich fair sind, Vertrauen in die beteiligten Institutionen (Brockner \& Siegel, 1995). Zweitens deutet eine faire Behandlung auch auf Respekt für die Rechte und die Bedürfnisse des Verhandlungspartners hin und trägt so zu einer langfristigen Vertrauensbildung (Folger \& Konovsky, 1989) bei. Drittens ist es einleuchtend anzunehmen, dass die „Fairness“ der Unternehmensbewertung und die „Fairness“ der Verteilung der Unternehmensanteile zwischen Investor und Unternehmen, welche diese Studie betrachtet, für beide Partner sensible Aspekte sind, die ihre nachfolgende Beziehung beeinflussen. Viertens kann eine faire Behandlung Vertrauensgefühle schaffen, indem Ausbeutungsängste vermieden werden können (Folger \& Konovsky, 1989). Außerdem wird in der bisherigen Forschung die Wahrnehmung von Fairness mit kooperativem Verhalten in Verbindung gebracht (Moorman, Niehoff \& Organ, 1993; Konovsky \& Pugh, 1994). Daher lauten Hypothesen vier und fünf:

Hypothese 4: Je höher die Fairness der Vertragsverhandlung in der vor-vertraglichen Phase ist, desto höher ist das Goodwill-Vertrauen in der nach-vertraglichen Phase.

Hypothese 5: Je höher die Fairness der Vertragsverhandlung in der vor-vertraglichen Phase ist, desto höher ist das Kompetenz-Vertrauen in der nach-vertraglichen Phase.

Offenheit der Due Diligence. In ihrem theoretischen Bezugssystem argumentieren Shepherd und Zacharakis (2001), dass Offenheit und Transparenz zur Vertrauensbildung zwischen Unternehmern und EKG beitragen. Größere Offenheit und Transparenz erhöhen den Informationsaustausch und die Qualität der Kommunikation auch über beidseitige Erwartungen in der nach-vertraglichen Phase und ermöglichen daher die Entstehung einer soliden Vertrauensbasis. Ebenfalls aus früherer Forschung ist bekannt, dass Offenheit und Transparenz in der organisatorischen Entscheidungsfindung bewiese- 
nermaßen einen positiven Einfluss auf eine Vielzahl von Gefühls- und Verhaltensreaktionen wie z.B. Vertrauen (Tyler \& Bies, 1990) besitzen. Zusammenfassend argumentiert diese Studie daher, dass ein hohes Maß an Offenheit und Transparenz in den vor-vertraglichen Interaktionen zu einem höheren Ausmaß sowohl an Goodwill- als auch an Kompetenz-Vertrauen in der späteren Zusammenarbeit zwischen EKG und Unternehmern führen wird. Dementsprechend folgen Hypothesen sechs und sieben:

Hypothese 6: Je höher die Offenheit der Due Diligence in der vor-vertraglichen Phase ist, desto höher ist das Goodwill-Vertrauen in der nach-vertraglichen Phase.

Hypothese 7: Je höher die Offenheit der Due Diligence in der vor-vertraglichen Phase ist, desto höher ist das Kompetenz-Vertrauen in der nach-vertraglichen Phase.

\section{Daten und Methoden}

\section{Untersuchungsdesign und Datenerhebung}

Diese Studie hat mit Hilfe eines Fragebogens im Zeitraum von Oktober 2001 bis Januar 2002 Daten von Venture-Capital-finanzierten Unternehmen erhoben ${ }^{8}$. Um einen hohen Anteil an zuverlässigen Antworten zu erreichen, wurden die Fragebögen im Rahmen der Haupterhebung an die CEOs und/oder die Gründer sämtlicher Unternehmen geschickt, welche zum Zeitpunkt der Erhebung den Selektionskriterien entsprachen. Analog zu den Vorschlägen von Vanderwerf und Brush (1989) wurden die Unternehmen nach folgenden Kriterien ausgewählt: Die Unternehmen mussten jünger als zehn Jahre alt sein (Covin, Slevin \& Covin, 1990), das Gründungsmanagement musste immer noch im Unternehmen tätig sein (Robinson, 1999) und die Unternehmen mussten ihren Firmensitz im deutschsprachigen Europa haben (Österreich, Deutschland, Liechtenstein, Luxemburg, Schweiz). Da es keine umfassende Datenbank Venture-Capital-finanzierter Unternehmen im deutschsprachigen Europa gibt, basiert die empirische Untersuchung auf einer selbst erstellten Datenbank, welche als ausreichend repräsentativ betrachtet werden kann, da sie keine systematischen Verzerrungen der in ihr enthaltenen Unternehmen aufweist (Bortz \& Döring, 1995: 452). Zahlreiche unterschiedliche Quellen wurden zur Erstellung der Datenbank verwendet, um alle relevanten Venture-Capital-Geber in den besagten Ländern zu erfassen: Die Jahresberichte des Bundesverband deutscher Kapitalgesellschaften (1995-2001), der Venture Capital Report - Guide to Venture Capital in Europe (1996), landesweite Businessplan-Wettbewerbe ${ }^{9}$, das BioRegio Netzwerk und die Kooperation mit anderen deutschsprachigen Forschern im selben Forschungsbereich.

Nach Erstellung der Datenbank wurden die Fragebögen direkt an CEOs und/oder Gründer von 1463 Venture-Capital-finanzierter-Unternehmen verschickt, welche fast die geschätzte Gesamtpopulation dieser Unternehmen zum Zeitpunkt der Studie darstellten ${ }^{10}$. CEOs und Gründer werden als die Informationsquellen mit dem besten und zuverlässigsten Wissen bezüglich der inter-organisationalen Beziehungen von jungen Unternehmen erachtet (so auch bei: Hambrick, 1981; Vanderwerf \& Brush, 1989; Glick, Huber, Miller, 
Doty \& Sutclif, 1990). Zur Beantwortung der Forschungsfragen war es zudem wichtig, dass die Befragten sowohl Auskunft über die allerersten Kontakte mit dem EKG als auch Auskunft zur Entwicklung der Zusammenarbeit bis zum Zeitpunkt der Befragung geben konnten. Für diese Anforderungen an die Befragten kommen nur Unternehmensgründer oder Mitglieder des Unternehmensvorstands in Frage, die bereits seit Gründung des Unternehmens in diesem tätig sind. Um die Validität der Antworten sicher zu stellen, wurde zusätzlich zur Vorauswahl der Fragebogenempfänger im Fragebogen selber durch eine Reihe von Kontrollfragen nochmals überprüft, ob die Befragten Unternehmensgründer und/oder Mitglieder des Vorstands sind, ob sie seit der Unternehmensgründung in die geschäftlichen Aktivitäten des Unternehmens involviert waren und ob sie Kenntnisse haben und Auskunft geben können über die ersten Interaktionen mit dem Venture-Capital-Geber des Unternehmens zum Zeitpunkt der Befragung. 78\% derjenigen, welche den Fragebogen ausgefüllt zurückgeschickt haben, waren Unternehmensgründer und gleichzeitig in der Geschäftsführung des Unternehmens tätig. Die restlichen 22\% waren Mitglieder der Geschäftsleitung, aber keine Unternehmensgründer. Insgesamt waren 95\% derjenigen, welche den Fragebogen ausgefüllt haben, Mitglieder der Unternehmensführung - also CEO, Geschäftsführer, Vorstandsmitglieder, Direktoren - und 5\% sonstige Mitarbeiter mit Führungsverantwortung, wie bspw. kaufmännischer Leiter. 100\% der Befragten gaben an, den derzeitigen EKG seit Beginn der ersten Zusammenarbeit zu kennen und gute Kenntnisse über die Zusammenarbeit mit dem EKG sowie die Entwicklung dieser Zusammenarbeit zu haben.

Für den postalischen Versand wurde das Erhebungsdesign von Dillman (1978) übernommen. Die Anzahl an vollständig ausgefüllten und verwertbaren Fragebögen beläuft sich auf 272, was einer insgesamt zufrieden stellenden Rücklaufquote von $20 \%$ entspricht. Die untersuchten Firmen lassen sich gemäß Selbstauskunft der befragten Firmen im Wesentlichen den folgenden Branchen zuordnen: 36\% Informationstechnologie, 26\% Life Sciences, 9\% Elektronik, 8\% Telekommunikation, 7\% Medien, 5\% Dienstleistungen, 4\% Maschinen- und Anlagenbau, 3\% Handel, 2\% Sonstige Branchen. Das durchschnittliche Alter der Firmen der Stichprobe beträgt 3,2 Jahre.

\section{Messgrößen}

Tabelle 1 gibt zudem Mittelwerte, Korrelationen und Werte für die Reliabilität der in der Studie verwendeten Konstrukte an, welche über dem geforderten Schwellenwert (Hair, Tatham, Anderson \& Black, 1998) von 0,70 (Cronbachs Alpha) liegen. Zur Überprüfung der Hypothesen wurden sowohl etablierte als auch neue Messgrößen verwendet. Die bereits bekannten Messgrößen wurden weitgehend aus Originalarbeiten übernommen. Wie Lewicki, Tomlinson und Gillespie (2006) anmerkten, hinkt die Messung von Vertrauen jedoch der theoretischen Entwicklung hinterher, weil bisher nicht genug Anstrengungen unternommen wurden, um die unterschiedlichen Dimensionen und Facetten von Vertrauen reliabel und valide zu messen. Auch Schoorman et al. (2007) stellten fest, dass die von ihnen entwickelten Messinstrumente für Vertrauen in einer Reihe von Studien eine für präzise Messungen zu niedrige Reliabilität von deutlich unter 0,70 (Cronbachs Alpha) aufgewiesen haben.

Die Konstruktion der Messinstrumente dieser Studie erfolgte nach folgendem Schema: Zuerst wurden - basierend auf einer ausführlichen Literaturrecherche zu den Dimensio- 
nen von Vertrauen - Definitionen der jeweiligen Konstrukte entwickelt. Für die Gewährleistung der inhaltlichen Validität wurden aufbauend auf diesen Definitionen deduktiv und induktiv eine Reihe von Items generiert, um die jeweiligen Konstrukte in einer umfassenden Art zu messen. In 16 Experteninterviews wurde diese Itemliste diskutiert und ergänzt. Nach einer Pilotstudie $(\mathrm{n}=30)$ wurde die Reliabilität der einzelnen Konstrukte überprüft, welche über dem Schwellenwert von Cronbachs Alpha 0,70 lag. Zusätzlich durchgeführte explorative Faktorenanalysen bestätigten zudem die Skalen-Zuordnung der Items auf die unterschiedlichen und konzeptionell vorgesehenen Konstrukte.

Abschnitt II 1 gibt einen Überblick zur Operationalisierung der Variablen. Mit Ausnahme der Kontrollvariablen handelt es sich bei den gemessenen Konstrukten um konstant wachsende Zustimmungsgrade zu Items auf einer siebenstufigen Skala. Die Messgrößen variieren dabei zwischen drei und fünf Items. Zur Überprüfung der Dimensionalität der Konstrukte wurden zunächst Hauptkomponentenanalysen durchgeführt. Anschließend wurden Reliabilitätsanalysen vorgenommen und die interne Konsistenz überprüft sowie die konvergente und diskriminante Validität der Skalen analysiert (Hair et al., 1998).

\section{Abhängige Variablen}

Goodwill-Vertrauen. (Quelle: Basierend auf Sako, 1992). Die Befragten wurden gebeten, auf einer siebenstufigen Skala anzugeben, ob der Investitionsmanager ihnen erlaubt, sogar in schwierigen Situationen ihren eigenen Weg zu gehen, ob der Investitionsmanager nicht Schwächen ihrerseits ausnutzt, ob Investitionsmanager und Unternehmer einander in hohem Maße vertrauen, ob der Investitionsmanager ihnen eher vertraut, als dass er sie kontrolliert. Cronbachs Alpha für diese Variable beträgt 0,75.

Kompetenz-Vertrauen. (Quelle: Basierend auf Sako, 1992). Das Konstrukt des „Kompetenz-Vertrauens" betrifft das Ausmaß, in welchem die Unternehmer den Investitionsmanager als erfahren in den für sie relevanten Bereichen einschätzen. Die Befragten wurden gebeten auf einer siebenstufigen Skala anzugeben, ob der Investitionsmanager Wissen über die Industrie, die Kunden, Produkte und Technologien sowie generell über die Gründung junger Unternehmen hat und ob er relevante Netzwerke und Kontakte besitzt. Cronbachs Alpha für diese Variable beträgt 0,86. Die Validität der Messung von Kompetenz-Vertrauen wird außerdem durch eine $0,58(\mathrm{p}<.001)$ bivariate Korrelation mit einem Außenkriterium - des Alters des EKG - gestützt, welches durch eine Datenbankabfrage ermittelt wurde. Firmenalter ist in der bisherigen Venture-Capital-Forschung schon mehrfach als Proxy für Reputation und Kompetenz von EKG verwendet worden (vgl. bspw. De Clercq, Sapienza \& Zaheer, 2003). Eine weitere Analyse zeigt zudem, dass diejenigen Unternehmer, welche den gleichen EKG hatten, in ihrer Einschätzung des Kompetenz-Vertrauens signifikant näher zusammen lagen als der Durchschnitt, was für die Validität der Messung des Kompetenz-Vertrauens spricht.

\section{Unabhängige Variablen}

Bedarfsanalyse. (Quelle: Basierend auf Ward, Duray, Leong \& Sum, 1995; Madhok \& Tallman, 1998). Die Befragten bewerteten auf siebenstufigen Skalen die Intensität der vor-vertraglichen Diskussion zwischen EKG und Unternehmen über die Risiken und 
Schwächen der jungen Unternehmen, die Notwendigkeit der Unterstützung und Beratung der Unternehmen und die Zusammenarbeit zwischen Unternehmen und EKG. Diese drei Antworten wurden zu einer zusammengesetzten Variable der „Bedarfsanalyse“ zusammengefasst. Cronbachs Alpha hierfür beträgt 0,79.

Fairness des Vertrags. (Quelle: Basierend auf Landström et al., 1998). Dieses Konstrukt gehört zu dem Konzept der verteilenden Gerechtigkeit und der Fairness von Verteilungsprozessen (Cobb et al., 1995). Das Konstrukt „Fairness des Vertrags“ basiert auf einer Studie von Landström et al. (1998) und wurde im Rahmen dieser Studie an die vorInvestitionsphase angepasst. Jeder Befragte gab an, wie großzügig die Verhandlungen waren, wie fair die Verhandlungen waren und wie leicht es war, eine Vereinbarung mit dem EKG zu treffen sowie, ob die Unternehmensbewertung als fair eingeschätzt wurde. Die vier Antworten wurden zu einer zusammengesetzten Variable der ,wahrgenommenen Fairness des Vertrags“" kombiniert. Cronbachs Alpha beträgt 0,73.

Offenheit der Due Diligence. Die Befragten wurden gebeten, auf einer siebenstufigen Skala Informationen über die Offenheit, Transparenz und Genauigkeit der Due Diligence zu liefern. Diese drei Items wurden zu einer Variable der „Offenheit der Due Diligence“ kombiniert. Cronbachs Alpha beträgt 0,73.

\section{Kontrollvariablen}

Anteil des Investors. Frühere Forschung zeigt, dass EKG oft umfangreiche Kontrollrechte in Unternehmen besitzen, in welche sie investieren (Fried, Bruton \& Hisrich, 1998; Sahlman 1990). Die Trennung zwischen Eigentum und Kontrolle ist eine potentielle Quelle für Zielkonflikte in kooperativen Beziehungen (Jensen \& Meckling, 1976; Milgrom \& Roberts, 1992). Ebenfalls bekannt ist, dass derartige Konflikte die Vertrauensbildung zwischen EKG und Unternehmern sehr stark beeinflussen können (Lind \& Tyler, 1988). Daher wurde in dieser Studie der Anteil des Hauptinvestors am Unternehmen als eine Kontrollvariable einbezogen. Der Mittelwert dieser Variable (gemessen in Prozent) beträgt 20,5 mit einer Standardabweichung von 15,5.

Einziges Angebot. Frühere Forschung über kontrafaktisches Denken ${ }^{11}$ definiert sich als der Versuch, einen Aspekt der Vergangenheit mental zu interpretieren durch die Vorstellung darüber, wie es hätte sein können, und so alternative Versionen der Vergangenheit (die gewisse Konsequenzen für die Gegenwart oder Zukunft haben könnten) zu ersinnen (Baron, 2000). Die bisherige Forschung zeigt zudem, dass kontrafaktisches Denken mit der Anzahl der tatsächlich zur Verfügung gestandenen Alternativen zusammen hängt (Baron, 2000). Es ist daher plausibel anzunehmen, dass die Möglichkeit für Unternehmer, zwischen unterschiedlichen Finanzierungsangeboten wählen zu können, die Wahrnehmung der Fairness des Vertrags beeinflussen kann. Im Kontext dieser Studie ist es daher relevant, ob den Unternehmen nur ein einziges Finanzierungsangebot vorgelegen hat oder ob sie zwischen mehreren Angeboten auswählen konnten. Die Befragten gaben im Fragebogen an, ob der vorliegende Hauptinvestor der einzige Investor war, der ein Finanzierungsangebot unterbreitete. Das Ergebnis zeigte, dass $81 \%$ der Unternehmen ein Angebot von mehr als einem EKG erhalten hatten.

Anzahl der Finanzierungsrunden. Frühere Forschung zeigt, dass sich Vertrauen mit der Zeit entwickelt, da die in die Beziehung involvierten Partner Erfahrungen im Umgang 
miteinander sammeln, sich gegenseitig kennen lernen und erfahren, wie viel sie von der anderen Seite erwarten können (Lewicki \& Bunker, 1995). Daher wird die Häufigkeit persönlicher Kontakte als wichtiger Einflussfaktor für die Bildung von Vertrauen angesehen. Mit einer Erhöhung der Anzahl der Kontakte können beide Seiten Informationen leichter austauschen und das Verhalten der anderen Seite besser vorhersagen, da sie mehr Zeit in unterschiedlichen Situationen miteinander verbringen konnten (Doney \& Cannon, 1997). Der Mittelwert dieser Variable beträgt 1,57 mit einer Standardabweichung von 1,1.

Top-Management-Team-Größe (TMT-Größe). Die Größe der Unternehmensleitung kann das Vertrauen der Unternehmer in die Kompetenz und den Goodwill ihrer EKG beeinflussen, da empirische Untersuchungen zeigen, dass größere Gruppen effektiver sind als kleinere Gruppen und bessere Problemlösungsfähigkeiten besitzen (Haleblian \& Finkelstein, 1993), was für die beim Investor wahrgenommene Kompetenz relevant sein kann. Größere Teams sollen zudem bessere Informationsverarbeitungs- und Entscheidungsfindungsfähigkeiten besitzen als kleinere Teams (Hambrick \& D'Avenil, 1992), was ebenfalls eine potentielle Einflussgröße auf das Kompetenzvertrauen der Unternehmer in ihre EKG sein kann. Der Mittelwert dieser Variable beträgt 2,9 mit einer Standardabweichung von 4,0 .

Erfahrung der Gründer. Die bisherige Forschung hat die Industrie- und Gründungserfahrung der Gründer von Unternehmen als zentrale Variable in der Zusammenarbeit zwischen EKG und Unternehmern untersucht (bspw. Gerpott \& Schefczyk, 2001b), und nach Sapienza und Gupta (1994) wird Vertrauen von der bisherigen Gründungserfahrung der CEOs oder Gründer von Unternehmen beeinflusst. Barney, Busenitz, Fiet und Moesel (1996) zeigen, dass die Erfahrung eines Gründungsteams die Wahrnehmung der Wertschöpfung des EKG beeinflussen kann, was sich möglicherweise wiederum auf die Wahrnehmung des Kompetenz-Vertrauens (und eventuell des Goodwill-Vertrauens) der Unternehmer in die EKG auswirkt. Die Befragten wurden daher gebeten, die Gesamtzahl an Management- und Industriejahren der Vorstandsmitglieder des Unternehmens anzugeben. Im Durchschnitt besaß der Vorstand 20,56 (kumulierte) Jahre an Erfahrung in Industrie und Management mit einer Standardabweichung von 19,1.

Unternehmensalter. Es ist plausibel anzunehmen, dass Unternehmen, die bereits seit einigen Jahren existieren, vertrauter mit den geschriebenen und ungeschriebenen Gesetzen ihrer eigenen Industrie sowie dem Bereich der Beteiligungsfinanzierung sind (Finkelstein \& Hambrick, 1996), da sie mehr Zeit hatten, diese Dinge zu lernen. Dieses größere Wissen beeinflusst möglicherweise die Bewertung der Kompetenz und der Vertrauenswürdigkeit der EKG und wird daher ebenfalls in dieser Studie kontrolliert. Der Mittelwert dieser Variable beträgt 42 Monate (3,5 Jahre) mit einer Standardabweichung von 37 (3,1 Jahre).

Hightech. In Hightech-Unternehmen, in denen eher technisches Wissen als Managerwissen eine Kernkompetenz der Unternehmer darstellt, könnten Kompetenzen und Fachwissen der EKG anders eingeschätzt werden (Sapienza, Korsgaard, Goulet \& Hoogendam, 2000) als in Low-Tech-Unternehmen. Daher wird in dieser Studie die Branche der Unternehmen kontrolliert.

Unternehmensphase. Mehrere Arbeiten haben bisher gezeigt, dass der Wertschöpfungsbeitrag von EKG je nach Unternehmensphase unterschiedlich ist. Da es sehr jungen Unternehmen an erfolgsrelevanten Erfahrungen, Kenntnissen und Fähigkeiten mangelt 
(Sapienza, 1992), und da in solchen Unternehmen das Team der neuen Firma noch wenig gemeinsame Arbeitserfahrung besitzt (Barney et al., 1996), wurde die Unternehmensphase, welches durch Selbsteinschätzung der Befragten auf einer dichotomen Skala erhoben wurde, auch als Kontrollvariable einbezogen, um Goodwill- und Kompetenz-Vertrauen vorherzusagen.

\section{E. Ergebnisse}

Tabelle 1 zeigt die Korrelationsmatrix sowie für jede Variable Mittelwert, Standardabweichung, Minimum und Maximum sowie Cronbachs Alpha für die gemessenen Konstrukte. Alle Werte für Cronbachs Alpha liegen über dem üblicherweise empfohlenen Schwellenwert für 0,70 (Hair et al., 1998). Die Korrelationen zwischen den unabhängigen Variablen liegen unter 0,35 und damit unter dem geforderten Schwellenwert von 0,50 (Hair et al., 1998). Um zu prüfen, ob Multikollinearität vorliegt, wurden bei den durchgeführten Regressionen sowohl die Varianzinflation als auch der Konditionsindex berechnet. Die Werte der Varianzinflation für alle Variablen liegen unter der Empfehlung von 1,3 und somit weit unter dem als Schwellenwert geltenden Wert von 10 für die Varianzinflation. Die Werte für den Konditionsindex (Hair et al., 1998) liegen zwischen 1 und 11,3. Ein Konditionsindex ist eine Zusammenfassung der Kollinearitätsdiagnose. Eine allgemein akzeptierte Regel zur Interpretation des Konditionsindex ist, dass Werte über 15 eine mögliche Kollinearität anzeigen und Werte über 30 eine ernste Kollinearität anzeigen. Die Ergebnisse der durchgeführten Analysen legen insgesamt nahe, dass Multikollinearität in der vorliegenden Studie kein Problem ist.

Eine Betrachtung von Tabelle 1 zeigt, dass auf bivariatem Niveau ein signifikanter Zusammenhang in der vermuteten Richtung zwischen Goodwill-Vertrauen und zwei unabhängigen Variablen (Fairness des Vertrags und Offenheit der Due Diligence) sowie zwischen Kompetenz-Vertrauen und allen drei unabhängigen Variablen (Bedarfsanalyse, Fairness des Vertrags und der Offenheit der Due Diligence) vorhanden ist. Tabelle 1 macht außerdem deutlich, dass es zwischen Goodwill-Vertrauen und Kompetenz-Vertrauen einen signifikanten positiven Zusammenhang $(\mathrm{r}=0,36, \mathrm{p}<.001)$ gibt. Hypothese eins kann demnach bestätigt werden.

Zur Überprüfung der Hypothesen zwei bis sieben wurde eine hierarchische Regressionsanalyse durchgeführt. Modell 1 der Tabelle 2 zeigt die Ergebnisse für Goodwill-Vertrauen, wenn lediglich die Kontrollvariablen (Anteil des Investors, Einziges Angebot, Anzahl der Finanzierungsrunden, TMT-Größe, Erfahrung der Gründer, Unternehmensalter, Hightech, Unternehmensphase) einbezogen werden. Der Anteil des Investors und der Erhalt von nur einem Angebot eines EKG zeigen als einzige Kontrollvariablen einen signifikanten, und zwar negativen Einfluss auf Goodwill-Vertrauen. Je höher der Anteil des Investors am Unternehmen, desto geringer ist das Goodwill-Vertrauen der Unternehmer in ihn. Diejenigen Unternehmer, welche mehrere Angebote zur Finanzierung hatten, haben ein geringeres Goodwill-Vertrauen in ihren EKG. Die Dummy-Variablen, welche eingeführt wurden, um die Finanzierungsphase des Unternehmens zu kontrollieren, sind hingegen in keiner der vier Regressionsgleichungen statistisch signifikant. Es spielt also für die Ergebnisse der Hypothesentests dieser Studie keine Rolle, ob die Unternehmen 


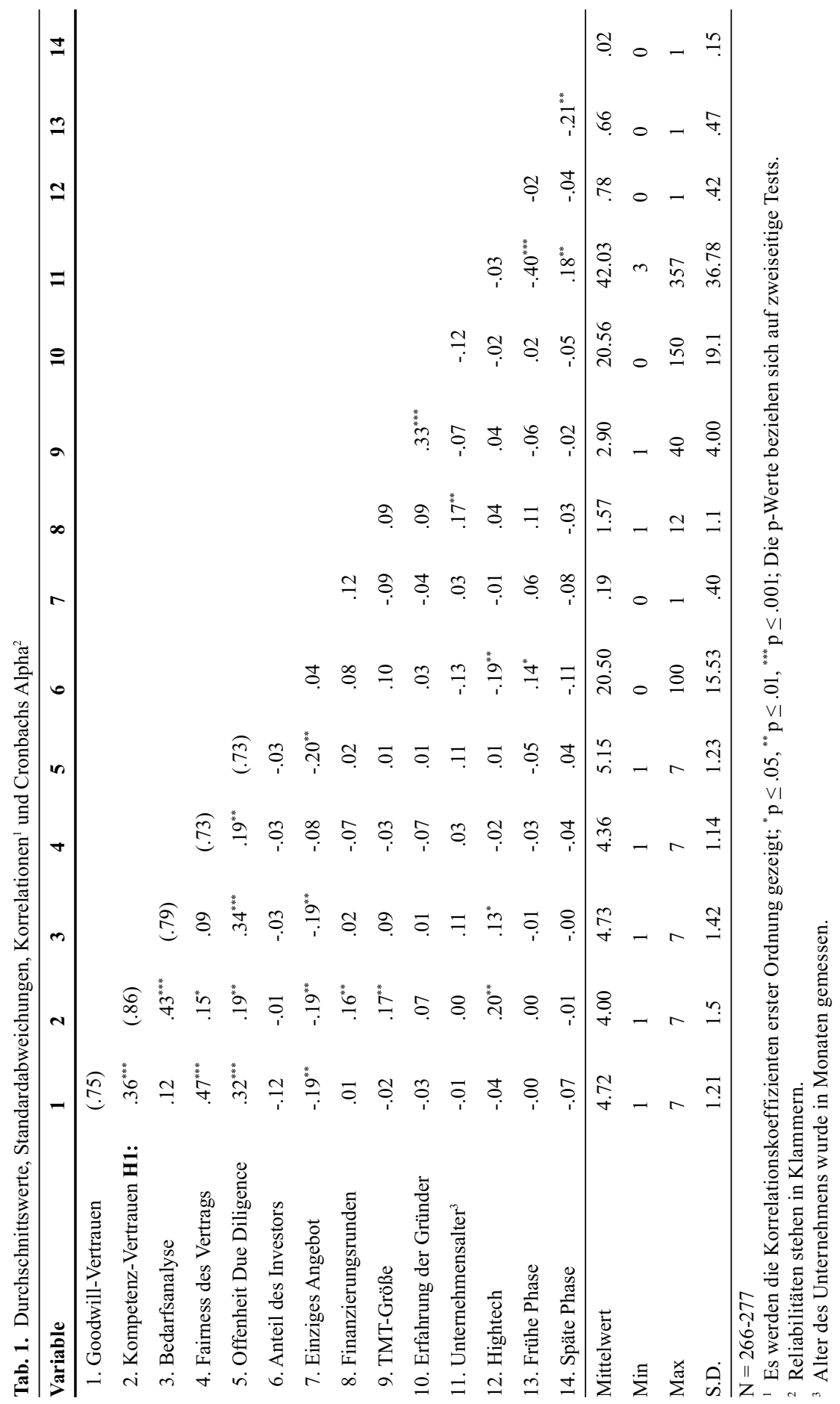


Tab. 2. Resultate der Regressionsanalysen ${ }^{1}$ für Goodwill-Vertrauen und Kompetenz-Vertrauen

\begin{tabular}{|c|c|c|c|c|}
\hline \multirow{3}{*}{ Unabhängige Variablen } & \multicolumn{4}{|c|}{ Abhängige Variablen } \\
\hline & \multicolumn{2}{|c|}{ Goodwill-Vertrauen } & \multicolumn{2}{|c|}{ Kompetenz-Vertrauen } \\
\hline & 1 & 2 & & 4 \\
\hline \multicolumn{5}{|l|}{ Haupteffekte } \\
\hline Bedarfsanalyse & H2: & -.00 & H3: & $.36^{* * *}$ \\
\hline Fairness des Vertrags & H4: & $.37^{* * * *}$ & H5: & $.12^{*}$ \\
\hline Offenheit der Due Diligence & H6: & $.21^{* * *}$ & H7: & .03 \\
\hline
\end{tabular}

\section{Kontrollvariablen}

Anteil des Investors

$\begin{array}{cccc}-.13^{*} & -.12^{*} & -.00 & .00 \\ -.20^{* *} & -.13^{*} & -.17^{* *} & -.01 \\ .05 & .07 & .16^{* *} & .16^{* *} \\ -.02 & -.02 & .13 & .10 \\ -.04 & -.01 & .01 & .03 \\ -.03 & -.06 & -.00 & -.01 \\ -.09 & -.08 & .17^{* *} & .13^{*} \\ -.01 & -.01 & -.00 & .01 \\ -.08 & -.06 & -.00 & -.00\end{array}$

F-Wert

Korrigiertes $\mathrm{R}^{2}$

Veränderung im korrigierten $\mathrm{R}^{2}$

$.20^{* * *}$

${ }^{1}$ Gezeigt werden die standardisierten Beta-Koeffizienten. Alle vier Modelle werden auf der gleichen Datenbasis getestet (Fallzahl $\mathrm{n}=208) ;{ }^{*} \mathrm{p} \leq .05,{ }^{* *} \mathrm{p} \leq .01,{ }^{* * *} \mathrm{p} \leq .001$; Die $\mathrm{p}$-Werte beziehen sich auf zweiseitige Tests.

2 War der aktuelle Venture-Capital-Geber der einzige Investor, der Ihnen ein Finanzierungsangebot gemacht hat?

sich in einer frühen oder späten Finanzierungsphase befinden. Ebenfalls keine Rolle für die Ergebnisse spielte die Anzahl der Finanzierungsrunden, die Erfahrung der Gründer, das Unternehmensalter und ob das Unternehmen aus einer Hightech-Branche kommt. In Modell 2 werden die unabhängigen Variablen zur Regression hinzugefügt und es zeigt sich, dass bei ihrer Hinzunahme in die Regressionsgleichung weitere 20\% der Varianz in der abhängigen Variablen erklärt werden können $\left(\mathrm{R}^{2}\right.$-Veränderung $=0,20 ; \mathrm{p}<0,001$; F-Wert: 7,77).

Modell 3 der Tabelle 2 zeigt die Ergebnisse für das Kompetenz-Vertrauen, wenn lediglich die Kontrollvariablen in der Regressionsgleichung enthalten sind. Sowohl der Erhalt von lediglich einem Angebot des Hauptinvestors als auch die Anzahl der Finanzierungsrunden mit dem jetzigen Hauptinvestor besitzen einen leicht negativen und signifikanten Zusammenhang mit Kompetenz-Vertrauen, und die Hightech-Industrie besitzt einen positiven Zusammenhang mit Kompetenz-Vertrauen. Modell 4 zeigt, dass bei Hinzunahme 
der unabhängigen Variablen zur Gleichung weitere 14\% der Varianz in der abhängigen Variablen erklärt werden können $\left(\mathrm{R}^{2}\right.$-Veränderung $=0,14 ; \mathrm{p}<0,001 ; \mathrm{F}$-Wert: 7,59).

Im Gegensatz zu der Vermutung aus Hypothese zwei zeigen die Regressionsergebnisse, dass die Bedarfsanalyse keine signifikante Vorhersage des Goodwill-Vertrauens ermöglicht. Daher kann Hypothese zwei nicht bestätigt werden. Hingegen können die Hypothesen drei, vier, fünf und sechs bestätigt werden: Die Auswirkungen der Bedarfsanalyse auf Kompetenz-Vertrauen sind positiv und signifikant $(p<0,001)$; Hypothese drei ist somit bestätigt. Die Fairness des Vertrags besitzt einen positiven Zusammenhang sowohl mit Goodwill-Vertrauen $(\mathrm{p}<0,001)$ als auch mit Kompetenz-Vertrauen $(\mathrm{p}<0,05)$ wie in den Hypothesen vier und fünf vorhergesagt wurde, welche demnach bestätigt sind. Die Auswirkungen der Offenheit der Due Diligence auf Goodwill-Vertrauen sind positiv und signifikant $(p<0,001)$ und bekräftigen daher Hypothese sechs. Schließlich wurde kein signifikanter Zusammenhang zwischen Kompetenz-Vertrauen und der Offenheit der Due Diligence entdeckt, und Hypothese sieben muss daher abgelehnt werden.

\section{F. Diskussion}

Das Ziel dieser Studie war es, den Einfluss inhaltlicher und prozessualer Aspekte der frühen Interaktionen zwischen EKG und Unternehmern auf das Kompetenz- und Goodwill-Vertrauen der Unternehmer in ihre EKG zu untersuchen. Konkret betrachtet wurden im Zusammenhang mit beiden Vertrauensarten die Intensität der Bedarfsanalyse, die wahrgenommene Fairness des Vertrags und die Offenheit und Transparenz der Due Diligence. Wie die Hypothesentests zeigen, konnten insgesamt fünf von sieben Hypothesen bestätigt werden. In ihrer Gesamtbetrachtung zeigen die Ergebnisse, dass die unabhängigen Variablen unterschiedlichen Einfluss auf die Entstehung von Goodwill- und Kompetenz-Vertrauen der Unternehmer in die EKG haben: Inhaltliche und sachbezogene Aspekte einer Interaktion führen gemäß dieser Studie eher zur Entstehung von Kompetenz-Vertrauen und prozessorientierte und qualitative Aspekte einer Interaktion führen eher zur Entstehung von Goodwill-Vertrauen. So konnte kein signifikanter Zusammenhang zwischen der Bedarfsanalyse in der vor-vertraglichen Phase und Goodwill-Vertrauen sowie Offenheit in der vor-vertraglichen Phase und Kompetenz-Vertrauen gefunden werden. Ingesamt betrachtet ergibt sich somit folgendes Bild: Eine intensive inhaltliche Auseinandersetzung in der frühen Zusammenarbeit beeinflusst positiv die Entstehung von Kompetenz-Vertrauen, aber nicht die Entstehung von Goodwill-Vertrauen. Eine große Fairness und Offenheit in der frühen Zusammenarbeit beeinflusst dagegen positiv die Entstehung von Goodwill-Vertrauen, aber nicht die Entstehung von Kompetenz-Vertrauen.

Die Ergebnisse dieser Studie heben auch die Rolle von Fairness und von Transparenz in der Entstehung von Goodwill-Vertrauen hervor und bestätigen somit frühere Studien, welche diesen Zusammenhang im Kontext von Großunternehmen gefunden haben (Shore, 2004; Korsgaard et al., im Erscheinen). Im Gegensatz zur Vermutung in Hypothese zwei konnte kein Einfluss der Intensität der Bedarfsanalyse während der ersten Zusammenarbeit auf das Goodwill-Vertrauen gefunden werden. Eine mögliche Erklärung für diesen Befund ist, dass eine intensive Bedarfsanalyse im Rahmen der ersten Kontakte es den 
Unternehmern zwar erlaubt, die Kompetenzen der Investoren genauer zu beurteilen, es ihnen aber nicht unbedingt erlaubt, zusätzlich die Güte der Absichten der Investoren einzuschätzen.

Deutlich wird diese Interpretation auch an folgendem Beispiel: Unternehmer, welche die Risiken und Schwächen ihres Unternehmens ihren EKG während der ersten Begegnungen mitteilen, können schnell feststellen, ob dem EKG Lösungs- und Unterstützungsmöglichkeiten bewusst sind; gleichzeitig braucht es aber eine gewisse Zeit der gemeinsamen Arbeit, um festzustellen, ob der EKG seine Kompetenz wohlwollend und beiderseitig nutzenmaximierend einsetzen wird. Während die Bedarfsanalyse konkrete Kompetenzen des EKG offenbart, welche kognitiv bewertet werden können und bei denen es unwahrscheinlich ist, dass sie sich innerhalb der Zusammenarbeit verändern, basiert der Goodwill auf emotionalen Wahrnehmungen und Zurechnungen, die leicht von lediglich einer „falschen“ Bemerkung beeinflusst werden und sich über die Zeit hinweg leicht verändern können. Wahrgenommene Kompetenzen sind zeitstabiler als wahrgenommenes Wohlwollen, welches sich sehr schnell ändern kann. Aus dieser Perspektive können die Ergebnisse auch als ein Beispiel für die Unterschiede zwischen kognitiver und emotionaler Beurteilung von Kooperationspartnern angesehen werden. Diese Interpretation wäre konsistent mit früheren Untersuchungen zum Einfluss von emotionalem und kognitivem Vertrauen (Devon \& Kent, 2005) und mit Eberl (2004), welcher argumentiert, dass Vertrauen auf Basis emotionaler Verbindungen zwischen Interaktionspartnern entsteht.

Zusammenfassend betrachtet zeigen die Ergebnisse dieser Studie außerdem, dass das Vertrauen der Unternehmer in ihre EKG in der Tat in zwei unterschiedliche Dimensionen differenziert werden kann, nämlich Kompetenz- und Goodwill-Vertrauen. Die Ergebnisse legen insbesondere dar, dass Kompetenz- und Goodwill-Vertrauen zwar zusammenhängen, jedoch eigenständige und unabhängige Konstrukte sind. Dies wird deutlich durch die moderate Korrelation zwischen beiden Variablen $(\mathrm{r}=0,36, \mathrm{p}<0,001)$. Dass die gemeinsame Varianz der beiden Vertrauensdimensionen lediglich $18 \%$ beträgt, zeigt, dass es sich nicht um identische, sondern um eigenständige Konstrukte handelt. Dieses Ergebnis ist auch ein Hinweis darauf, dass die Befragten in der Lage waren, zwischen beiden Dimensionen ihres Vertrauens in den EKG zu differenzieren. Obwohl Kompetenz- und Goodwill-Vertrauen moderat zusammenhängen, liegen für beide Vertrauensdimensionen unterschiedliche Vorhersagestrukturen durch die unabhängigen Variablen vor. Relevant ist dieser Befund demnach auch für die theoretisch und konzeptionell vielfach diskutierte Unterscheidung zwischen Goodwill- und Kompetenz-Vertrauen. Die Ergebnisse bestätigen frühere konzeptionelle Studien, welche das Vertrauen in den Goodwill und das Vertrauen in die Kompetenz von Kooperationspartnern als unabhängig voneinander thematisiert haben (Sako, 1992; Das \& Teng, 2001; Gefen, 2002), und sie tragen zur Schaffung größerer konzeptioneller Klarheit im Bezug auf die unterschiedlichen Formen von Vertrauen und ihre inter-organisationalen Beziehungen bei, wie es auch von Welter und Smallbone (2006) gefordert wird.

Insgesamt weisen die Ergebnisse darauf hin, dass eine wesentlich detailliertere Sicht auf Vertrauen im Gründungskontext und in inter-organisationalen Beziehungen notwendig ist, als dies bislang in empirischen Studien der Fall war (vgl. bspw. McKnight, Cummings \& Chervany, 1998; Butler, 1999; Manigart et al., 2001; Blomqvist, 2002). Die Unterschiede im Beziehungsmuster zwischen Vertrauensarten und Wirkungsvariablen unter- 
streichen die Notwendigkeit, zukünftig nicht nur das Niveau, sondern auch die Art des Vertrauens zu betrachten. Die Unterscheidung beider Vertrauensdimensionen - Kompetenz- und Goodwill-Vertrauen - ermöglicht es, komplexe Kooperationsmechanismen in Kontexten wie demjenigen junger Unternehmen zu entflechten und zu verstehen.

Die vorliegende Studie trägt auf unterschiedliche Weise zum aktuellen Stand der Literatur in verschiedenen Forschungsgebieten bei: Erstens verbessern die Ergebnisse den aktuellen Wissenstand zu Einflussfaktoren auf zwei unterschiedliche Dimensionen des Vertrauens unter besonderer Berücksichtigung der frühen Phase einer kooperativen Beziehung. Zweitens tragen die Ergebnisse zum Forschungsgebiet der Beziehung zwischen Eigenkapital-Gebern und jungen Unternehmen bei und zwar aus der bisher selten berücksichtigten Perspektive der Unternehmensgründer. Darüber hinaus adressiert die Studie durch Einbeziehung der vor-vertraglichen Phase bisher „weiße Flecken“ auf der Forschungslandkarte der Gründungs- und insbesondere der Venture-Capital-Forschung, wie von Landström et al. (1990) gefordert. Drittens baut sie auf den theoretisch-konzeptionellen Arbeiten von Cable und Shane (1997), De Clercq und Sapienza (2001) sowie Shepherd und Zacharakis (2001) auf, welche eine Vielzahl an Thesen bezüglich der Rolle von Vertrauen in der Kooperation zwischen EKG und Unternehmern aufführen. Viertens bestätigen die Ergebnisse frühere Arbeiten über unterschiedliche Vertrauensdimensionen (bspw. Sako, 1992), indem sie zwei distinkte Vertrauensdimensionen empirisch bestätigen. Schließlich untersuchte die Studie beide Vertrauensarten in einem spezifischen Kontext, welcher durch hohe Unsicherheit, Risiko und Informationsasymmetrie gekennzeichnet ist und welcher für beide Akteure mit hohen Austrittskosten aus der Zusammenarbeit verbunden ist. Die Ergebnisse haben daher Aussagekraft auch über den unmittelbaren Kontext der Gründungsforschung hinaus für kooperative Beziehungen, welche unter ähnlichen Umständen entstehen und aufrecht erhalten werden wie bspw. bei der Finanzierung von wissenschaftlichen oder industriellen Forschungs- und Entwicklungsprojekten.

Die Ergebnisse der Studie haben auch Implikationen für die Praxis. Sie verdeutlichen Unternehmern und EKG, dass bereits die erste Begegnung und die ersten Interaktionen für die Qualität einer Zusammenarbeit große Bedeutung haben und den Erfolg von Kooperationsbeziehungen beeinflussen können. Sie verdeutlichen, dass eine Kooperationsbeziehung faktisch bereits vor der formalen Unterzeichnung eines Kooperationsvertrags beginnt, und betonen die Rolle von Fairness, Transparenz und der inhaltlichen Auseinandersetzung der zukünftigen Zusammenarbeit auch in der vor-vertraglichen Phase. Weiter belegen die Ergebnisse, dass insbesondere eine tiefgehende Bedarfsanalyse den Unternehmern dazu dienen kann, die Kompetenzen der Investoren zu erkennen und helfen kann, die richtigen Investoren zu finden. Es kann daher sowohl Venture-Capital-Firmen als auch Gründern empfohlen werden, auf einer derartigen inhaltlichen Auseinandersetzung, welche Risiken, Schwächen und die Form der künftigen Zusammenarbeit zum Inhalt hat, vor Vertragsunterzeichnung zu bestehen.

Ähnlich wie andere Studien besitzt auch diese Untersuchung Einschränkungen und Grenzen, welche hinsichtlich der Interpretation und Generalisierbarkeit der Ergebnisse beachtet werden müssen. Die mit der gewählten Methode der Fragebogenerhebung verbundenen Einschränkungen wurden bereits in Abschnitt D III thematisiert. Zusätzlich zu der Überprüfung, ob sich systematische Verzerrungen in den Daten finden, wurden - wo es möglich war - Korrelationen der Konstrukte mit objektiv erhobenen Außenkriterien 
durchgeführt. Obwohl die in Abschnitt D III beschriebenen Tests insgesamt nicht auf eine Verzerrung der Daten hindeuten, ist diese bei Fragebogenerhebungen nie mit absoluter Sicherheit auszuschließen (Kaya, 2006), und Zusammenhänge aus nicht-experimentellen oder nicht-längsschnittlichen Forschungsdesigns können daher nur mit Vorbehalt kausal interpretiert werden (z.B. Biddle \& Marlin, 1987; Cliff, 1983).

Immer wenn Fragestellungen untersucht werden, für die es nicht im ausreichendem Maße archivierte Daten gibt - wie es für die vorliegende Studie der Fall ist - müssen Wissenschaftler auf subjektive Befragungen der beteiligten Personen zurückgreifen (Kumar, Stern \& Anderson, 1993). Eine subjektive Bewertung wäre aber auch unabhängig vom Vorhandensein objektiver Daten für diese Studie nötig, weil aufgrund der Fragestellung und Hypothesen alleine die Beurteilung durch die Gründer und Vorstandsmitglieder die Gelegenheit bietet, Informationen über subjektiv erlebte und subjektiv wahrgenommene mehrdimensionale Variablen der Beziehung zwischen EKG und Unternehmern ${ }^{12} \mathrm{zu}$ erfahren (Dess \& Robinson, 1984). Auch Welter und Smallbone (2006) weisen darauf hin, dass Vertrauen subjektiv empfunden wird und kaum „objektiv“ gemessen werden kann.

Die Befragten mussten im Rahmen der Beantwortung sowohl Angaben zu den zurückliegenden ersten Interaktionen mit dem EKG machen als auch Angaben zur aktuellen Zusammenarbeit mit dem EKG. Die Gefahr einer Datenverzerrung durch Retrospektion ist aber vor allem dann gegeben, wenn die Befragten aufgefordert sind, dasselbe Konstrukt einmal rückblickend und einmal aktuell einzuschätzen (Huber \& Power, 1985), da die retrospektive Einschätzung die aktuelle Einschätzung beeinflusst. Die Gefahr einer retrospektiven Verzerrung der Antworten ist in dieser Studie daher als geringer zu erachten $^{13}$. Für die Befragung wurden entsprechend den Vorschlägen von Huber und Power (1985) diejenigen Personen ausgewählt, welche das größte Wissen im Bezug auf die Forschungsfragen hatten: nämlich die Unternehmensgründer und/oder Mitglieder der Geschäftsführung, welche von Beginn des Unternehmens an in seine Entwicklung involviert waren. Frühere Studien haben allerdings auf die Gefahr einer systematischen Verzerrung bei der Befragung von nur einer Person pro Unternehmen hingewiesen (Kumar, Stern \& Anderson, 1993; Ernst, 2003), welche bis zu 30\% der gesamten Varianz ausmachen und die Konstruktvalidität gefährden kann (z.B. Ernst, 2003).

Die Gefahr eines Informant Bias in der vorliegenden Studie wird zum einen durch den Untersuchungskontext junger Unternehmen und zum anderen durch die Konstruktion des Erhebungsinstrumentes gemindert ${ }^{14}$.

Die Stichprobe, welche dieser Studie zugrunde liegt, ist beschränkt auf Venture-Capital-finanzierte Firmen und damit auf technologieintensive Wachstumsunternehmen. Diese Einschränkung der Stichprobe auf Venture-Capital-finanzierte Unternehmen ist aber auch als eine Stärke der vorliegenden Studie zu verstehen: Erstens erlaubt sie eine kontextspezifische Betrachtung organisationstheoretischer Prozesse, wie wiederholt von anderen Wissenschaftlern gefordert (vgl. Johns, 2001; Rousseau \& Fried, 2001). Bei der Untersuchung von Vertrauen argumentieren einige Forscher, dass es wichtig ist, externe, situationsbedingte und soziale Aspekte voll einzubeziehen, anstatt Vertrauen als individuelles Verhalten oder Element der Persönlichkeit zu betrachten (Rotter, 1971). Nach Lindskold und Bennet (1973) und Mayer, Davis und Schoorman (1995) stellt Vertrauen ein psychologisches Konzept dar, welches auf sozialen Interaktionen in der Umwelt, in der die 
Beziehung entsteht, basiert. Den Zusammenhang wegzulassen, verursacht unvermeidbar eine vereinfachende Reduktion, welche es nicht erlaubt, die tatsächlichen Elemente des Phänomens Vertrauen zu erfassen (Lewis \& Weigert, 1985). Auch im Bereich Entrepreneurship haben frühere Studien auf die Bedeutung des Kontextes für die untersuchten Fragestellungen hingewiesen und vor einer Unterschätzung externer Faktoren gewarnt (bspw. Davidsson, 2003). Genauer gesagt ermöglicht die Fokussierung dieser Studie auf den Kontext von technologieintensiven Wachstumsunternehmen die Untersuchung der Entstehung von Vertrauen in einem Umfeld, das durch große Unsicherheit, hohes Risiko, eine hohe Gefahr opportunistischen Verhaltens der Kooperationspartner bei hohem finanziellem (bzw. persönlichem) Einsatz, in welchem keine vollständigen Verträge zur Absicherung dieser Risiken abgeschlossen werden können, geprägt ist. Es mag plausibel erscheinen, dass aufgrund der Eigenschaften dieses Kontexts die Verletzung von Kompetenz-Vertrauen ähnlich schwerwiegend ist wie die Verletzung von Goodwill-Vertrauen in anderen Kontexten und dass sowohl Kompetenz-Vertrauen als auch Goodwill-Vertrauen für eine Kooperation zwischen EKG und Unternehmen vorhanden sein müssen. Darüber hinaus wirkt die Beschränkung der Stichprobe als zusätzliche Kontrolle der Entstehungs- und Wachstumsbedingungen der Firmen (Lechner, Dowling \& Welpe, 2006) und trägt so zur Homogenisierung der Stichprobe bei, wodurch potentielle Verzerrungen aufgrund industrieübergreifender Analysen verhindert werden können (Baum, Calabrese \& Silverman, 2000).

Die Ergebnisse dieser Studie müssen unter Beachtung der oben aufgeführten Einschränkungen und Grenzen interpretiert werden. Gleichzeitig bieten sie jedoch Anhaltspunkte für zukünftige Untersuchungen. Zukünftige Studien könnten den Befund dieser Studie, dass die Bedarfsanalyse mit höherem Kompetenz-Vertrauen in die Investoren in Verbindung steht, dahingehend überprüfen, ob die eigentliche Erfüllung der Bedürfnisse des Unternehmens oder nur das Ausmaß, in welchem der EKG versucht, diese Bedürfnisse zu erfüllen, das Kompetenz-Vertrauen in den EKG beeinflusst. Zweitens könnten zukünftige Studien aus Sichtweise junger Unternehmen die Folgen von sowohl Goodwill- als auch Kompetenz-Vertrauen betrachten. Es ist plausibel anzunehmen, dass Goodwill- und Kompetenz-Vertrauen einen jeweils spezifischen Einfluss bspw. auf Konfliktsituationen, Entscheidungen über die Entwicklung des Unternehmens sowie den Unternehmenserfolg haben. Aktuelle Forschungsarbeiten weisen darauf hin, dass Vertrauen in Abhängigkeit von der Phase der Unternehmensentwicklung sowohl negative als auch positive Folgen haben und unter Umständen kontraproduktiv in inter-organisationalen Beziehungen sein kann (Goel \& Karri, 2006; Welter \& Smallbone, 2006; Zahra et al., 2006). Zukünftige Studien könnten daher die Kosten und den Nutzen von Goodwill- und Kompetenz-Vertrauen im Verlauf inter-organisationaler Beziehungen betrachten. Ebenfalls denkbar ist, dass beide Vertrauensdimensionen unterschiedlichen moderierenden Einflüssen unterworfen sind. Mellewigt, Madhok und Weibel (2007) zeigen bspw., dass Vertrauen sowohl als Substitut für Kontrollmechanismen als auch als Komplement für Koordinationsbestrebungen dienen kann. Weitere Forschungsvorhaben könnten im Anschluss an Mellewigt, Madhok und Weibel (2007) und diese Studie untersuchen, welches die moderierenden Rollen beider Vertrauensdimensionen sind. McKnight et al. (1998) stellen eine Reihe von Hypothesen zu 'initial trust formation' auf. Es wäre in zukünftigen Studien interessant zu prüfen, welche Faktoren mit der Entstehung von 'initial' GoodwillVertrauen bzw. 'initial' Kompetenz-Vertrauen zusammenhängen ${ }^{15}$. Ebenfalls interessant 
wäre es, in zukünftigen Studien neben Goodwill- und Kompetenz-Vertrauen zusätzlich noch Integritäts-Vertrauen als dritte Dimension, wie von Mayer et al. (1995) vorgeschlagen, zu untersuchen ${ }^{16}$.

Diese Studie betrachtete lediglich Venture-Capital-finanzierte Unternehmen. Die Anwendung des Modells auf andere Zusammenhänge wie beispielsweise Familienunternehmen oder wachstumsschwache Unternehmen würde ein interessantes und fruchtbares Forschungsfeld darstellen. Die Prädiktoren von Vertrauen in der vorliegenden Studie stammen aus der vor-vertraglichen Phase. Eine Vielzahl anderer Variablen, vor allem aus der nach-vertraglichen Phase, wie z.B. die Ähnlichkeit zwischen EKG und Unternehmen, die Kommunikationsstrukturen (vgl. bspw. Fallgatter \& Koch, 1998) die Motivation und Anstrengungen des EKG und die Art und Anzahl der auftretenden Konflikte zwischen EKG und Unternehmen, könnten einen Einfluss oder Zusammenhang auf die Vertrauensbildung besitzen. Abschließend betrachtet liefern die Ergebnisse dieser Studie Antworten zu Fragen über die Beschaffenheit von Vertrauen in inter-organisationalen Beziehungen und ergänzen so frühere Forschungsarbeiten über die Vertrauensbildung in Partnerschaften (Das \& Teng, 1998; McKnight, Cummings \& Chervany, 1998). Insgesamt stellt diese Studie einen ersten Versuch dar, die Dynamik der Vertrauensbildung in einem unternehmerischen Zusammenhang aufzuzeigen. Indem belegt werden konnte, dass unterschiedliche Bedingungen die Entstehung von Goodwill- und Kompetenz-Vertrauen beeinflussen, erfüllt diese Studie auch Forderungen der Gründungsforschung nach Aufklärung der Entstehung von Vertrauen im unternehmerischen Umfeld und unter besonderer Berücksichtigung der vor-vertraglichen Phase.

\section{Appendix}

\section{Goodwill-Vertrauen}

Bitte geben Sie auf einer Skala von 1 bis 7 Ihre Zustimmung oder Ablehnung zu den folgenden Aussagen an:

"Der Leadinvestor lässt uns auch in schwierigen Situationen unseren eigenen Weg gehen"

"Der Leadinvestor nutzt Schwächen unsererseits in Verhandlungen nicht aus"

"Der Leadinvestor und wir vertrauen uns gegenseitig in großem Ausmaß"

"Der Leadinvestor vertraut uns statt uns zu kontrollieren"

\section{Kompetenz-Vertrauen}

Bitte geben Sie auf einer Skala von 1 bis 7 Ihre Zustimmung oder Ablehnung zu den folgenden Aussagen an:

"Der Leadinvestor hat exzellente Kenntnisse über unsere Industrie"

"Der Leadinvestor hat exzellente Kenntnisse über unsere Kunden"

"Der Leadinvestor hat exzellente Kenntnisse über unsere Produkte und Technologie"

"Der Leadinvestor hat exzellente Kenntnisse über Unternehmensgründungen"

"Der Leadinvestor hat exzellente Netzwerke und Kontakte" 


\section{Bedarfsanalyse}

Bitte geben Sie auf einer Skala von 1 bis 7 Ihre Zustimmung oder Ablehnung zu den folgenden Aussagen an:

"Der Leadinvestor und wir haben vor Vertragsunterzeichnung intensiv über unsere zukünftige Zusammenarbeit und Kooperation gesprochen"

"Der Leadinvestor und wir haben vor Vertragsunterzeichnung intensiv über die Risken und Schwächen des Unternehmens gesprochen"

"Der Leadinvestor und wir haben vor Vertragsunterzeichnung intensiv über unseren Bedarf an Unterstützung und nicht-finanzielle Leistungen gesprochen "

\section{Fairness des Vertrags}

Bitte geben Sie auf einer Skala von 1 bis 7 Ihre Zustimmung oder Ablehnung zu den folgenden Aussagen an:

"Die Vertragsverhandlungen waren fair"

"Die Unternehmensbewertung war fair"

"Es war leicht, sich mit dem Leadinvestor auf die Vertragsbedingungen zu einigen"

\section{Offenheit der Due Diligence}

Bitte geben Sie auf einer Skala von 1 bis 7 Ihre Zustimmung oder Ablehnung zu den folgenden Aussagen an:

"Die Due Diligence mit dem Leadinvestor war offen"

"Die Due Diligence mit dem Leadinvestor war transparent"

"Die Due Diligence mit dem Leadinvestor war exakt"

\section{Anmerkungen}

1 Bspw. haben Welter und Smallbone (2006) ein Sonderheft der Zeitschrift Entrepreneurship Theory and Practice zum Thema Vertrauen herausgegeben.

2 Vgl. für die Bedeutung der Kontextualisierung empirischer Fragestellungen organisationstheoretischer Forschung Rousseau \& Fried (2001) und die Bedeutung der Kontextualisierung empirischer Fragestellungen im Entrepreneurship-Bereich Davdisson (2003).

3 Die Betreuung durch den VC-Geber in der Postinvestment Phase lässt sich in drei Gebiete aufteilen (Manigart et al., 2001), nämlich die Tiefe und Art der Betreuung, die Rollen die VCG spielen in der Beratung und Betreuung ihrer Firmen und die Ergebnisse und Erfolg der jeweiligen Betreuung. Den Gründern fehlen jedoch häufig die notwendigen Managementqualifikationen, da viele Gründer einen technisch-naturwissenschaftlichen Hintergrund haben und daher beim Aufbau, der Organisation und Führung eines Unternehmens auf externe Unterstützung angewiesen sind. Nur wenige Gründer in Deutschland können Gründungserfahrungen vorweisen. In der Start-up Phase erwarten und brauchen die Gründer mehr als in allen anderen Unternehmensphasen die Unterstützung ihrer VCG. Daraus ergibt sich ein hoher Bedarf nach externer Managementunterstützung in allen unternehmerischen Fragestellungen mit einer sich im Entwicklungsverlauf verändernden Bedarfsstruktur und die Notwendigkeit, diese Defizite durch geeignete Beratung und Unterstützung auszugleichen. Ansonsten würde die Unternehmensentwicklung behindert oder könnte scheitern. Forschungsergebnisse weisen VCG als die wichtigsten Partner junger Unternehmen aus für die komplementäre Deckung fehlender Ressourcen und als Mentoren und Coaches (Sapienza, 1989).

'By supplying expertise the venture capitalists can help the venture overcome the liabilities of newness'. (Sapienza \& Amason, 1993) 
4 Im weiteren Verlauf der Arbeit wird der Begriff „Goodwill“ weiter verwendet, da sich der Begriff „Goodwill-Trust“ in der einschlägigen Literatur bereits etabliert hat und eine sinnerhaltende Übersetzung ins Deutsche mit nur einem Wort nicht möglich ist.

5 Beispielsweise unterscheidet Sako (1992) zwischen „competence trust“ (ist der andere Verhandlungspartner fähig, das zu tun, was er vorgibt zu tun?) und ,goowill trust“ (wird der Verhandlungspartner eine unbefristete Verpflichtung mit dem Ziel beidseitiger Nutzenmaximierung eingehen, ohne unfaire Vorteile für sich selber herauszuschlagen?).

6 Im Gegensatz zu Goodwill-Vertrauen, welches bisher häufig im Zentrum von Untersuchungen zu Vertrauen stand, ist Kompetenz-Vertrauen bisher nur selten Gegenstand empirischer Studien gewesen.

7 Due Diligence bezeichnet die „Prüfung der Beteiligungswürdigkeit von und Verhandlung mit potentiellen Portfoliounternehmen“ (Schefczyk, 2000: 38). Im Rahmen der Due Diligence wird u.a. die Managementkompetenz, das Marktpotential, die Produktdifferenzierung, die Wettbewerbsposition, der Geschäftsplan und der Zeithorizont des Unternehmens geprüft (Schefczyk, 2000).

8 Im Rahmen dieser Studie wurde eine Eigenerhebung gewählt, weil Sekundärdaten zu den für die Beantwortung der Forschungsfrage notwendigen Variablen nicht erhältlich sind (vgl. für ein ähnliches Vorgehen bspw. Davidsson \& Wiklund, 2000; Lyon, Lumpkin \& Dess, 2000). Fragebogenerhebungen sind vor allem dann geboten, wenn andere Quellen nicht zugänglich sind, wie dies regelmäßig bei Untersuchungen über junge und nicht-öffentliche Unternehmen (Dess \& Robinson, 1984; Chandler \& Hanks, 1993) und so auch im Rahmen dieser Studie der Fall ist. Die frühere Forschung unterstützt jedoch die Verlässlichkeit und Validität von selbst erhobenen Daten im Unternehmensgründungskontext (Sapienza 1989; Brush \& Vanderwerf, 1992; Orpen, 1993; Stubner, Wulf \& Hungenberg, 2007). Der Fragebogen dieser Studie wurde vor der Hauptbefragung einem ausführlichen Pre-Test unterzogen.

9 Für die Identifikation junger Unternehmen wurden folgenden Businessplanwettbewerbe einbezogen: Münchener Business Plan Wettbewerb, Businessplan-Wettbewerb Nordbayern, Businessplan-Wettbewerb BerlinBrandenburg, Start-up in Bayern, Businessplanwettbewerb Sachsen-Anhalt; NUK - Neues Unternehmertum Rheinland e.v., Businessplan Wettbewerb Liechtenstein, Science4Life / Der Businessplan-Wettbewerb, Thüringer Businessplan Wettbewerb, Berliner Businessplan-Wettbewerb, Businessplan-Wettbewerb Startsocial.

10 Die Annahme, dass mit 1463 identifizierten Venture-Capital-finanzierten Unternehmen gemäß konservativer Schätzung mindestens 90\% derjenigen Unternehmen, welche zum Zeitpunkt der Befragung die Stichprobenselektionskriterien erfüllten, aufgenommen wurden, deckt sich auch mit der Untersuchung und Analyse der Zeitschrift „Finance Magazin“ (2002) sowie einer weiteren Schätzung, welche vom BVK (Bundesverband Deutscher Kapitalgesellschaften) 2002 veröffentlicht wurde.

11 Beim kontrafaktischen Denken gibt es sowohl eine ins Negative gekehrte Ausprägung „,andere Umstände wären besser als die zutreffenden“ als auch eine ins Positive gekehrte Ausprägung ,,andere Umstände wären schlechter als die zutreffenden".

12 Die Fragen in der empirischen Erhebung bezogen sich auf den Leadinvestor und nicht auf den einzelnen Investmentmanager. Es ist denkbar, dass in der vor-vertraglichen Phase andere Personen in engem Kontakt standen als in der nachvertraglichen Phase. Entscheidend sind jedoch die ,subjektiven' Eindrücke, welche die Unternehmer von ihren Investoren vor und nach Vertrag haben.

13 Es wurden jeweils unterschiedliche Konstrukte für die Vergangenheit und Gegenwart abgefragt wurden (Morgenstern \& Barrett, 1974). Darüber hinaus waren die Fragen, welche sich auf die vor-vertragliche Phase und die nach-vertragliche Vertrauenseinschätzung bezogen, grafisch deutlich voneinander getrennt. Auch in der Anleitung zum Ausfüllen des Fragebogens wurde explizit darauf hingewiesen, dass sich der erste Teil des Fragebogens auf die Qualität und den Inhalt der Zusammenarbeit mit dem EKG vor Vertragsabschluss bezieht und der zweite Teil des Fragebogens auf das zum Zeitpunkt der Erhebung aktuelle Kompetenz- und Goodwill-Vertrauen. Hinzu kommt außerdem, dass in dieser Studie kein langer Rückerinnerungszeitraum für die Befragten notwendig war. Im Durchschnitt lag der Vertragsschluss mit dem Investor zum Zeitpunkt der Befragung nur 1,8 Jahre zurück. Erinnerungszeiträume von bis zu 2 Jahren haben nachgewiesenermaßen noch eine ausreichend hohe Validität (Ericsson \& Simon, 1980). Zudem ist es bekannt, dass vor allem sich wiederholende Ereignisse bei restrospektiver Einschätzung der Gefahr der Verzerrung ausgesetzt sind (Huber \& Power, 1985). Die Verhandlungen mit dem Hauptinvestor dürften jedoch für die große Mehrzahl der Unternehmen ein einschneidendes und einmaliges Erlebnis gewesen sein und deshalb einer Erinnerung gut zugänglich sein.

14 Ein Informant Bias tritt vor allem aufgrund unterschiedlicher funktionaler und hierarchischer Positionen von Informanten im Unternehmen auf (Ernst, 2003). Unternehmensgründer haben aber sowohl die gleiche hierarchische Position als auch sehr ähnliche funktionale Aufgaben (Clarysse \& Moray, 2004). Wahrnehmungsunterschiede, welche zwischen Informanten aufgrund unterschiedlicher Hierarchieebenen entstehen kön- 
nen, sind im Gründungskontext nicht gegeben. Darüber hinaus zeigt West (2007), dass Entscheidungen, welche neu gegründete Unternehmen betreffen, zumeist kollektive Teamentscheidungen sind. Der Informant Bias tritt auch dann auf, wenn Informanten unterschiedlich vertraute und bekannte Informationen in ihren Urteilen heranziehen (Ernst, 2003). Auch hier gilt, dass im Gründungskontext - anders als in Großunternehmen - die Mitglieder der Geschäftsführung und insbesondere die Unternehmensgründer gleichen Zugang zu Informationen haben (West, 2007). Selbst bei einer frühen funktionalen Spezialisierung der Gründer auf unterschiedliche Aufgaben ist aus der Gründungsforschung bekannt, dass die Mitglieder der Geschäftsführung sowohl Aufgaben außerhalb ihres formalen Aufgabenbereiches wahrnehmen als auch über Informationen, welche das Unternehmen allgemein betreffen, informiert sind (West, 2007). Ein weiterer Einflussfaktor auf den Informant Bias ist eine unterschiedlich lange Zugehörigkeit zum Unternehmen. In der vorliegenden Befragung wurde jedoch sicher gestellt, dass die Befragten seit Gründung des Unternehmens in dessen Entwicklung involviert waren. Weiter ist die Gefahr eines Informant Bias auch dann gegeben, wenn die befragten Personen aufgefordert sind, generalisierbare Aussagen über ,patterns of behaviour, after summarizing either observed (actual) or expected (prescribed) organizational relations" (Seidler, 1974: 817) zu machen. In der vorliegenden Studie machten die Befragten jedoch Angaben zu ihren persönlichen Einstellungen, Meinungen und Verhaltensweisen und ihrer subjektiven Einschätzung bezüglich des Goodwill- und Kompetenz-Vertrauens. Insgesamt identifizieren Huber \& Power (1985) vier Hauptgründe, warum Informanten in Befragungen falsche oder verzerrte Angaben machen: Sie haben erstens Anreize, dies zu tun, zweitens sind ihre Wahrnehmungs- oder Informationsverarbeitungsfähigkeiten beschränkt, drittens haben sie kein Wissen über die abgefragten Sachverhalte und viertens sind sie mit unangemessenen Erhebungsinstrumenten befragt worden. Im Rahmen dieser Studie wurden mehrere Maßnahmen getroffen, um Falschangaben oder die Verzerrung von Angaben aufgrund der oben genannten Gründe zu minimieren: Die Befragung erfolgte anonym, um ein sozial erwünschtes Antwortverhalten zu vermeiden und um den Befragten die Angst vor persönlichen Auswirkungen oder Auswirkungen für das Unternehmen zu nehmen. Im Fragebogen wurde zudem (gemäß den Vorschlägen von Seidler, 1974) mit einer Reihe von Kontrollfragen überprüft, ob die Befragten entweder Gründer oder Mitglied der Geschäftsleitung des Unternehmens sind, ob sie seit Beginn der Zusammenarbeit mit dem EKG im Unternehmen tätig waren, ob sie vertiefte Kenntnisse über den Beginn und der Entwicklung der Beziehung zum EKG haben. Um die Motivation der Befragten zu erhöhen, wurde zudem vor Versenden der Fragebögen ein Informationsblatt, welches auf die Nützlichkeit der Angaben für den Erkenntnisgewinn der Organisations- und Gründungsforschung hinwies, an die Befragten versendet. Kincaid und Bright (1957) zeigen, dass Nützlichkeit und Relevanz des durch die Befragung erlangten Erkenntnisgewinns einen starken Anreiz für eine aufmerksame Teilnahme für Mitglieder der Unternehmensführung ausübt.

15 Dieser Hinweis stammt von einem anonymen Gutachter.

16 Dieser Hinweis stammt von einem anonymen Gutachter.

\section{Literatur}

Albach, H. (1980): Vertrauen in der Ökonomischen Theorie. Zeitschrift für die gesamte Staatswissenschaft, 136(1): $2-11$.

Aldrich, H. \& Auster, E.R. (1986): Even dwarfs started small: liabilities of age and size and their strategic implications. L.L. Cummings \& B.M. Staw (Hrsg.): Research in Organizational Behavior, 8. Greenwich, CT, JAI Press: $165-198$.

Amaeshi, K. \& Scarbrough, H. (2006): Does trust matter? Exploring the link between investment decisions and trustworthiness in knowledge markets. EBK Working Paper.

Amit, R., Glosten, L. \& Muller, E. (1990): Entrepreneurial ability, venture investments, and risk sharing. Management Science, 36(10): 1232-1245.

Axelrod, R. \& Dijon, D. (1988): The further evolution of cooperation. Science, 242: 1385-1390.

Ba, S. \& Pavlou, P.A. (2002): Evidence of the effect of trust building technology in electronic markets: Price premiums and buyer behavior. MIS Quarterly, 26(3): 243-268.

Barney, J.B. \& Hansen, M.H. (1994): Trustworthiness: Can it be a source of competitive advantage? Strategic Management Journal, 15(2): 175-203.

Barney, J.B., Busenitz, L., Fiet, J.O. \& Moesel, D. (1989): The structure of venture capital governance: An organizational economic analysis of relations between venture capital firms and new ventures. Academy of Management Proceedings: 64-68. 
Baron, R.A. (2000): Counterfactual thinking and venture formation - The psychology of new venture creation. Journal of Business Venturing, 15(1): 79-91.

Baum, J., Calabrese, T. \& Silverman, B. (2000): Don't go it alone: alliance network composition and startups' performance in Canadian biotechnology. Strategic Management Journal 21(3): 267-294.

Biddle B.J. \& Marlin M.M. (1987): Casualty, confirmation, credulity and structural equation modeling. Child Development, 58: 4-17.

Blomqvist, K. (2002): Partnering in the dynamic environment - the role of trust in asymmetric partnership formation. Thesis for the degree of doctor of science. Lappeenranta University of Technology, Finland.

Bortz, J. \& Döring, N. (1995): Forschungsmethoden und Evaluation. Zweite Auflage, Berlin: Springer.

Borys, B. \& Jemison, D. (1989): Hybrid arrangements as strategic alliances: Theoretical issues in organization combinations. Academy of Management Review, 14: 234-329.

Brettel, M., Thust, S. \& Witt, P. (2001): Die Beziehung zwischen VC-Gesellschaften und Start-Up-Unternehmen. Working Paper, Wissenschaftliche Hochschule für Unternehmensführung (WHU), Vallendar.

Breuer, W., Antonczyk, R. \& Mark, K. (2007): Covenants in venture capital contracts : Theory and empirical evidence from the German capital market. In G.N. Gregoriou, M. Kooli \& R. Kräussl (Hrsg.): Venture Capital in Europe. North Holland, Elsevier: 233-247.

Brockner, J. \& Siegel, P. (1995): Understanding the interaction between procedural and distributive justice: The role of trust. In R.M. Kramer \& T.R. Tyler (Hrsg.): Trust in organizations: Frontiers of theory and research. Thousand Oaks, CA: Sage: 391-413.

Brush, C. \& Vanderwerf, P. (1992): A comparison of methods and sources for obtaining estimates of new venture performance. Journal of Business Venturing, 7: 157-170.

Bundesverband Deutscher Kapitalbeteiligungsgesellschaften - German Venture Capital Association e.V. BVK, (1995, Hrsg.): BVK Statistik. Berlin: BVK.

Bundesverband Deutscher Kapitalbeteiligungsgesellschaften - German Venture Capital Association e.V. BVK (1996, Hrsg.): BVK Statistik 1996. Berlin: BVK.

Bundesverband Deutscher Kapitalbeteiligungsgesellschaften - German Venture Capital Association e.V. BVK (1997, Hrsg.): BVK Statistik 1997. Berlin: BVK.

Bundesverband Deutscher Kapitalbeteiligungsgesellschaften - German Venture Capital Association e.V. BVK (1998, Hrsg.): BVK Statistik 1998. Berlin: BVK.

Bundesverband Deutscher Kapitalbeteiligungsgesellschaften - German Venture Capital Association e.V. BVK (1999, Hrsg.): BVK Statistik 1999. Berlin: BVK.

Bundesverband Deutscher Kapitalbeteiligungsgesellschaften - German Venture Capital Association e.V. BVK (2000, Hrsg.): BVK Statistik 2000. Berlin: BVK.

Bundesverband Deutscher Kapitalbeteiligungsgesellschaften - German Venture Capital Association e.V. BVK (2001, Hrsg.): BVK Statistik 2001. Berlin: BVK.

Bundesverband Deutscher Kapitalbeteiligungsgesellschaften - German Venture Capital Association e.V. BVK (2002, Hrsg.): BVK Statistik 2002. Berlin: BVK.

Bunduchi, R. (2005): Business relationships in Internet based electronic markets: The role of goodwill trust and transaction costs. Information Systems Journal, 15(4): 321-341.

Busenitz, L., Fiet, J.O. \& Moesel, D. (2004): Reconsidering the venture capitalists' 'value added' proposition: An international learning perspective. Journal of Business Venturing, 19: 787-807

Butler, J. (1999): Trust expectations, information sharing, climate of trust, and negotiation effectiveness and efficiency. Group and Organization Management, 24(2): 217-238.

Cable, D.M. \& Shane, S. (1997): A prisoner's dilemma approach to entrepreneur-venture capitalist relationships. Academy of Management Review, 22(1): 142-176.

Chan, Y.S., Siegel, D. \& Thakor, A.V. (1990): Learning, corporate control and performance requirements in venture capital contracts. International Economic Review, 31(2): 365-382.

Chandler, G. \& Hanks, S. (1993): Measuring the performance of emerging businesses: a validation study. Journal of Business Venturing, 8: 391-408.

Clark, M.S. \& Mills, J. (1979): Interpersonal attraction in exchange and communal relationships. Journal of Personality and Social Psychology, 37(1): 12-24.

Clark, M.S., Mills, J. \& Powell, M.C. (1986): Keeping track of needs in communal and exchange relationships. Journal of Personality and Social Psychology, 51: 333-338.

Clark, M.S. \& Waddell, B. (1985): Perceptions of exploitation in communal and exchange relationships. Journal of Social and Personal Relationships, 2: 403-418.

Clarysse, B. \& Moray, N. (2004): A process study of entrepreneurial team formation: The case of a research spin-off. Journal of Business Venturing, 19: 55-79. 
Cliff, N. (1983): Some cautions concerning the application of casual modeling methods. Multivariate Behavioral Research, 18: 115-126.

Cobb, A.T., Wooten, K.C. \& Folger, R. (1995): Justice in the making: Toward understanding the theory and practice of justice in organizational change and development. Research in Organizational Change and Development, 8: 243-295.

Covin, J., Slevin, D. \& Covin, T. (1990): Content and performance of growth-seeking strategies: a comparison of small firms in high- and low-technology industries. Journal of Business Venturing, 5: 391-412.

Damasio, A. (2005): Human behaviour: Brain trust. Nature, 435(7042): 571-572.

Das, T.K. \& Teng, B.-S. (1998): Between trust and control: developing confidence in partner cooperation in alliances. Academy of Management Review, 23(3): 491-512.

Das, T.K. \& Teng, B.-S. (2001): Trust, control, and risk in strategic alliances: An integrated framework. Organization Studies, 22(2): 251-283.

Davidsson, P. (2003): The domain of entrepreneurship research: Some suggestions. In J. Katz \& D. Shepherd (Hrsg.): Cognitive approaches advances in entrepreneurship, firm emergence and growth. Oxford: Elsevier/ JAI Press: 315-372.

Davidsson, P. \& Wiklund, J. (2000): Conceptual and empirical challenges in the study of firm growth. In: D. Sexton \& H. Landström. (Hrsg.): Handbook of Entrepreneurship, Oxford: Blackwell Publishing: 26-44.

De Clercq, D. \& Sapienza, H.J. (2001): The creation of relational rents in venture capitalist-entrepreneur dyads. Venture Capital, 3(2): 107-128.

De Clercq D., Sapienza H.J. \& Zaheer A. (2003): Freeriding the coattails? Effects of effort and reputation on venture capital firm value added. Academy of Management Meetings, Entrepreneurship Division, August, Seattle, USA.

Demougin, D. \& Fabel, O. (2007): Entrepreneurship and the division of ownership in new ventures. Journal of Economics \& Management Strategy, 16(1): 111-128.

Dess, G.G. \& Robinson Jr., R.B. (1984): Measuring organizational performance in the absence of objective measures: The case of the privately-held firm and conglomerate business unit. Strategic Management Journal, 5: 265-273.

Devon, J. \& Kent, G. (2005): Cognitive and affective trust in service relationships. Journal of Business Research, 58(4): 500-507.

Dillman, D.A. (1978): Mail and telephone surveys: the total design method. New York: John Wiley \& Sons.

Dirks, K.T. \& Ferrin, D.L. (2002): Trust in leadership: Meta-analytic findings and implications for organizational research. Journal of Applied Psychology, 87(4): 611-628.

Doney, P.M. \& Cannon, J.P. (1997): An examination of the nature of trust in buyer-seller relationships. Journal of Marketing, 4: 35-51.

Eberl, P. (2004): The development of trust and implications for organizational design: a game- and attributiontheoretical-framework. Schmalenbach Business Review, 56(3): 258-273.

Ericsson, K.A. \& Simon, H.A. (1980): Verbal reports as data. Psychological Review, 87: 215-251.

Ernst, H. (2003): Ursachen eines Informant Bias und dessen Auswirkung auf die Validität empirischer betriebswirtschaftlicher Forschung. Zeitschrift für Betriebswirtschaft, 73(12): 1249-1275.

Fallgatter, M.J. \& Koch, L.T. (1998): Kooperationen im Spannungsfeld konstruierter Wirklichkeiten: Zur Entstehung einer betrieblichen Kooperation. In. E. Spieß \& F.W. Nerdinger (Hrsg.): Kooperation in Unternehmen. München, Mering: Hampp: 13-32.

Ferrin, D.L. Dirks, K.T. \& Shah, P.P. (2006): Direct and indirect effects of third-party relationships on interpersonal trust. Journal of Applied Psychology, 91(4), 870-883.

Fiedler M. \& Welpe, I.M. (2006): Venture capital investors and the development of nanotechnology ventures. Working paper. Munich School of Management, Munich.

Finance Magazin - Das Finanzmagazin für Unternehmer (2002): Existenzgründung \& Venture Capital, Mai: 18-24.

Finkelstein, S. \& Hambrick, D. (1996): Top executives and their effects on organizations. St. Paul, MN: West Publishing Company.

Folger, R. \& Konovsky, M. (1989): Effects of procedural and distributive justice on reactions to pay raise decisions. Academy of Management Journal, 32: 15-130.

Fried, V.H., Bruton, G. \& Hisrich, R. (1998): The involvement of the board of directors in portfolio company strategy. Journal of Private Equity, 1(4): 51-55.

Ganesan, S. (1994): Determinants of long-term orientation in buyer-seller relationships. Journal of Marketing, 58(April): 1-19.

Gefen, D. (2002): Reflections on the dimensions of trust and trustworthiness among online consumers. ACM SIGMIS Database, 33(3): 38-53. 
Gerpott, T.J. \& Schefczyk, M. (2001a): Management support for portfolio companies of venture capital firms: An empirical study of German Venture Capital Investments. British Journal of Management 12: 201-216.

Gerpott, T.J. \& Schefczyk, M. (2001b): Qualifications and turnover of managers and venture capital-financed firm performance. Journal of Business Venturing 16: 145-163.

Glick, W., Huber, G., Miller, C., Doty, D. \& Sutclif, K. (1990): Studying changes in organizational design and effectiveness: retrospective event histories and periodic assessments. Organization Science, 1: 293-312.

Goel, S. \& Karri, R. (2006): Entrepreneurs, effectual logic, and over-trust. Entrepreneurship: Theory \& Practice, 30(4): 477-493.

Green, R. (2003): Measuring goodwill trust between groups of people: Three years of an industry oil alliance. Strategic Change, 12: 367-379.

Grichnik. D. \& Immerthal, L. (2005): Rationalität und Risiko der Gründungsentscheidung. Die Betriebswirtschaft, 65(6): 563-583.

Güth, W., Ockenfels, P. \& Wendel, M. (1992): Cooperation based on trust - an experimental investigation. Journal of Economic Psychology, 18: 15-43.

Hair, J.F., Anderson, R.E., Tatham, R.L. \& Black, W.C. (1998): Multivariate data analysis. Fünfte Auflage, Upper Saddle River, New Jersey: Prentice Hall: 577-667

Haleblian, J. \& Finkelstein, S. (1993): Top management team size, CEO dominance, and firm performance: The moderating roles of environmental turbulence and discretion. Academy of Management Journal, 36(4): 844-863.

Hambrick, D. (1981): Environment, strategy and power within top management teams. Administrative Science Quarterly, 26: 253-276.

Hambrick, D.C. \& D'Aveni, R.A. (1992): Top team deterioration as part of the downward spiral of large corporate bankruptcies. Management Science, 38(10): 1445-1466.

Harrison, R.T., Dibben, M.R. \& Mason, C.M. (1997): The role of trust in the informal investment decision: an exploratory analysis. Entrepreneurship: Theory and Practice, 24(4): 63-81.

Hellmann, T. (1998): The allocation of control rights in venture capital contracts. RAND Journal of Economics, 29(1): $57-77$.

Hellmann, T. (2007): Entrepreneurs and the process of obtaining resources. Journal of Economics \& Management Strategy, 16(1): 81-109.

Hirschmann A. (1970): Exit, voice and loyalty - responses to decline in firms, organizations and states. Cambridge, MA: Harvard University Press.

Holmes, J.G. \& Rempel, J.K. (1989): Trust in close relationships. In C. Hendrick (Hrsg.): Close relationships: Review of Personality and Social Psychology, 10: 187-220.

Hommel, U., Farag, H., Witt, P. \& Wright, M. (2004): Contracting, monitoring and exiting venture investments in transitioning economies: A comparative analysis of Eastern European and German markets. Venture Capital: An International Journal of Entrepreneurial Finance, 6(4): 257-282.

Howorth, C. \& Moro, A. (2006): Trust within entrepreneur bank relationships: Insights from Italy. Entrepreneurship: Theory \& Practice, 30(4): 495-517.

Huber, G.P. \& Power, D.J. (1985): Retrospective reports of strategic managers: guidelines for increasing their accuracy. Strategic Management Journal, 6: 171-180.

Jensen, M.C. \& Meckling, W.H. (1976): Theory of the firm: Managerial behavior, agency costs and ownership structure. Journal of Financial Economics, 3(4): 303-360.

Johns, G. (2001): In praise of context. Journal of Organizational Behavior, 22: 31-42.

Jones, G.R. \& George, J.M. (1998): The experience and evolution of trust: Implications for cooperation and teamwork. Academy of Management Review, 23(3): 531-546.

Kaja, M. (2006): Verfahren der Datenerhebung. In: S. Albers, D. Klapper, U. Konradt, W. Walter \& J. Wolf (Hrsg.): Methoden der empirischen Forschung. Gabler: Wiesbaden: 55-72.

Kim, P.H., Ferrin, D.L., Cooper, C.D. \& Dirks, K.T. (2004): Removing the shadow of suspicion: The effects of apology versus denial for repairing competence - versus integrity-based trust violations. Journal of Applied Psychology, 89(1): 104-118.

Kincaid, H.V. \& Bright, M. (1957): Interviewing the business elites. American Journal of Sociology, 63(3): 304-311.

Kirilenko, A.A. (2001): Valuation and control in venture finance. Journal of Finance, 56(2): 565-597.

Kollmann, T. \& Kuckertz, A. (2006): Investor relations for start-ups: an analysis of venture capital investors' communicative needs. International Journal of Technology Management, 34: 47-62.

Konovsky, M.A. \& Pugh, D.S. (1994): Citizenship behaviour and social exchange. Academy of Management Journal, 37: 656-699. 
Korsgaard, M.A., Pitariu, H.A. \& Jeong, S.Y. (im Erscheinen): Trust in management: An interpersonal perspective. C.L. Cooper \& J. Barling (Hrsg.): Handbook of organizational behavior. Thousand Oaks, CA: Sage

Kosfeld, M., Heinrichs, M., Zak, P J., Fischbacher, U. \& Fehr, E. (2005): Oxytocin increases trust in humans. Nature, 435(2): 673-676.

Kumar, N., Stern, L. \& Anderson, J. (1993): Conducting interorganizational research using key informants. Academy of Management Journal, 36: 1633-1651.

Landström, H. (1990): Co-operation between venture capital companies and small firms. Entrepreneurship and Regional Development, 2(4): 345-362.

Landström, H., Manigart, S., Mason, C. \& Sapienza, H. (1998): Contracts between entrepreneurs and investors: Terms and negotiation processes. In P.D. Reynolds, W.D. Bygrave, N.M. Carter, S. Manigart, C.M. Mason, G.D. Meyer \& K.G. Shaver (Hrsg.): Frontiers of entrepreneurship research 1998. Wellesley, MA: Babson College: $571-585$.

Larson, A. (1992): Network dyads in entrepreneurial settings: A study of the governance of exchange relationships. Administrative Science Quarterly, 37(1): 76-104.

Lechner, C., Dowling, M. \& Welpe, I.M. (2006): Firm Networks and Firm Development: The Role of the Relational Mix. Journal of Business Venturing, 21(4): 514-540.

Lewicki, R.J., Tomlinson, E. C. \& Gillespie, N. (2006): Models of interpersonal trust development: theoretical approaches, empirical evidence, and future directions. Journal of Management, 32: 991-1022.

Lewicki, R.J. \& Bunker, B.B. (1995): Trust in relationships: A model of trust development and decline. In B.B. Bunker \& J.Z. Rubin (Hrsg.): Conflict, cooperation and justice, 133-173. San Francisco: Jossey-Bass.

Lewis, J.D. \& Weigert, A. (1985): Trust as a social reality. Social Forces, 63(4): 967-985.

Lind, E.A. \& Tyler, T. (1988): The social psychology of procedural justice. New York: Plenum.

Lindskold S. \& Bennet R. (1973): Attributing trust and conciliatory intent from coercive power capability. Journal of Personality and Social Psychology, 28(2): 180-186.

Lyon, D., Lumpkin, G. \& Dess, G. (2000): Enhancing entrepreneurial orientation research: operationalizing and measuring a key strategic decision making process. Journal of Management, 26: 1055-1085.

Macmillan, I.C., Kulow, D.M. \& Khoylian, R. (1989): Venture capitalists' involvement in their investments: Extent and performance. Journal of Business Venturing, 4(1): 27-47.

Madhok, A. \& Taliman, S. (1998): Resources, transactions and rents: Managing value through interfirm collaborative relationships. Organization Science, 9: 326-339.

Manigart, S., Korsgaard, A.M., Folger, R., Sapienza, H. \& Baeyens, K. (2001): Trust in private equity contracts. Vlerik Working Papers 2002/1.

Mayer, R.C., Davis, J.H. \& Schoorman, F.P. (1995): An integrative model of organizational trust. Academy of Management Review, 20(3): 709-734.

McEvily, B., Perrone, V. \& Zaheer, A. (2003): Trust as an organizing principle. Organization Science, 14(1): 91-103.

McKnight, D.H., Cummings, L.L. \& Chervany, N.L. (1998): Initial trust formation in new organizational relationships. Academy of Management Review, 23: 473-490.

Meier, D., Hiddemann, T. \& Brettel, M. (2006): Wertsteigerung bei Buyouts in der Post-Investment-Phase - der Beitrag von Private-Equitiy-Firmen zum operativen Erfolg ihrer Portfoliounternehmen im europäischen Vergleich. Zeitschrift für Betriebswirtschaft, 10(76): 1035-1066.

Mellewigt, T., Madhok, A. \& Weibel. A. (2007): Trust and formal contracts in interorganizational relationships substitutes and complements. Erhältlich zum Download im Social Science Citation Network: http://papers. ssrn.com/sol3/papers.cfm?abstract_id=611161.

Mesquita, L.F. (2007): Starting over when the bickering never ends: The role of trust. facilitators in cluster development. Academy of Management Review, 32(1): 72-91.

Milgrom, P.R. \& Roberts, J. (1992): Economics, Organization and Management. Englewood Cliffs.

Moorman, R., Niehoff, B. \& Organ, D. (1993): Treating employees fairly and organizational citizenship behavior: Sorting the effects of job satisfaction, organizational commitment, and procedural justice. Employee Responsibilities and Rights Journal, 6(3): 209-225.

Morgenstern, R. \& Barrett, N. (1974): The retrospective bias in unemployment reporting by sex, race and age. Journal of the American Statistical Association, 69(346): 355-357.

Morse, E.A., Fowler, Sally W. \& Lawrence, T.B. (2007): The impact of virtual embeddedness on new venture survival: Overcoming the liabilities of newness. Entrepreneurship: Theory \& Practice; 31(2): 139159.

Neergaard, H. \&Ulhøi, J.P. (2006): Government agency and trust in the formation and transformation of interorganizational entrepreneurial networks. Entrepreneurship: Theory \& Practice, 30(4): 519-539. 
Nguyen, T.V., Le, N.T. \& Freeman, N.J. (2006): Trust and uncertainty: A study of bank lending to private SMEs in Vietnam. Asia Pacific Business Review, 12(4): 547-568.

Orpen, C. (1993): Strategic planning, scanning activities and the financial performance of small firms. Journal of Small Business and Entrepreneurship, 11: 62-72.

Page, W.G. (2007): Collective cognition: When entrepreneurial teams, not individuals, make decisions. Entrepreneurship Theory and Practice, 31(1): 77-102.

Pavlou, P.A. (2002): Institution-based trust in interorganizational exchange relationships: the role of online B2B marketplaces on trust formation. Journal of Strategic Information Systems, 11: 215-243.

Pennings, J. \& Woiceshyn, J. (1987): A typology of organizational control and its metaphors. In: N. Ditomaso \& S.B. Bacharach (Hrsg.): Research in the sociology of organization. Greenwich, CT: JAI Press: 73-104.

Reißig-Thust, S., Brettel, M. \& Witt, P. (2004): Vertragsgestaltung durch Venture Capital-Gesellschaften, Finanz Betrieb, 6(9): 636-645.

Ring, P. \& Van de Ven, A. (1992): Structuring cooperative relationships between organizations. Strategic Management Journal 13(7): 483-498.

Robinson, K. (1999): An examination of the influence of industry structure on eight alternative measures of new venture performance for high potential independent new ventures. Journal of Business Venturing, 14: 165187.

Roese, N. (1997): Counterfactual thinking. Psychological Bulletin, 121(1): 133-148.

Rotter, J.B. (1971): Generalized expectancies for interpersonal trust. American Psychologist, 26: 443-452.

Rousseau, D.M. \& Fried, Y. (2001): Location, location, location: Contextualizing organisational research. Journal of Organizational Behavior, 22: 1-13.

Sahlman, W.A. (1990): The structure and governance of venture-capital organizations. Journal of Financial Economics, 27(2): 473-521.

Sako, M. (1992): Prices, quality and trust. Inter-firm relations in Britain and Japan. Cambridge: Cambridge University Press.

Saparito, P. (2000): Three dimensions of trust in bank-small firm relationships: instrument development and preliminary examination of the production of trust by various organizational structures. Working paper präsentiert auf der 20. Babson-Kauffmann Entrepreneurship Research conference, Wellesly, USA, Juni 8-10.

Saparito, P.A., Chen, Ch. C. \& Sapienza, H.J. (2004): The role of relational trust in small bank-firm relationships. Academy of Management Journal, 47(3): 400-411.

Sapienza, H.J. \& Korsgaard, M. (1996): The role of procedural justice in entrepreneur-venture capital relations. Academy of Management Journal, 39: 544-574.

Sapienza, H.J. \& Gupta, A.K. (1994): Impact of agency risks and task uncertainty on venture capitalist-entrepreneur relations. Academy of Management Journal, 37(6): 1618-1632.

Sapienza, H.J. (1989): Variations in venture capitalist-entrepreneur relations: antecedents and consequences. Unpublished doctoral dissertation, University of Maryland.

Sapienza, H.J. (1992): When do venture capitalists add value? Journal of Business Venturing, 7(1): 9-27.

Sapienza, H.J., Korsgaard, M.A., Goulet, P.K. \& Hoogendam, J.P. (2000): Effects of agency risks and procedural justice on board processes in venture capital-backed firms. Entrepreneurship \& Regional Development, 12(4): 331-351.

Schefczyk, M. (1999): Mehr als nur Geld: Notwendigkeit und Nutzen einer Beratungsunterstützung von Portfoliounternehmen durch Venture Capital-Gesellschaften. In: M. Berrios Amador, K. Lohmann \& F. Pleschak, (Hrsg.): Beteiligungskapital in der Unternehmensfinanzierung. Wiesbaden, Gabler: 131-152.

Schefczyk, M. (2000): Erfolgsstrategien deutscher Venture-Capital-Gesellschaften. Stuttgart: Schäffer-Poeschel, 2. Auflage.

Schefczyk, M. \& Gerpott, T. (1998): Beratungsunterstützung von Portfoliounternehmen durch deutsche VentureCapital-Gesellschaften. Eine empirische Untersuchung. Zeitschrift für Betriebswirtschaft, 2: 143-165.

Schoorman, F.D., Mayer, R.C. \& Davis, J.H. (2007): An integrative model of organizational trust: Past, present, and future. Academy of Management Review, 32(2): 344-354.

Seidler, J. (1974): On using informant's: A technique for collecting quantitative data and controlling for measurement error in organizational analysis. American Sociological Review, 39: 816-831.

Shepherd, D.A. \& Zacharakis, A. (2001): The venture capitalist-entrepreneur relationship: control, trust and confidence in co-operative behaviour. Venture Capital, 3(2): 129-150.

Shore, T. (2004): Equity sensitivity theory: do we all want more than we deserve? Journal: Journal of Managerial Psychology, 19(7): 722-728.

Smart, G.H. (1998): Management assessment methods in venture capital: Toward a theory of human capital valuation. Claremont, $\mathrm{CA}$. 
Steier, L. \& Greenwood, R. (1995): Venture capitalist relationships in the deal structuring and post-investment stages of new firm creation. Journal of Management Studies, 32(3): 337-359.

Stubner, S., Wulf, T. \& Hungenberg, H. (2007): Management support and the performance of entrepreneurial start-ups - an empirical analysis of newly founded companies in Germany. Schmalenbach Business Review, 59, 138-159.

Stuart, R. \& Abetti, P.A. (1987): Start-up ventures - towards the prediction of initial success. Journal of Business Venturing, 2: 215-230.

Timmons, J. \& Bygrave, W. (1986): Venture capital's role in financing innovation for economic growth. Journal of Business Venturing, 1: 161-176.

Tyler, T.R. \& Bies, R.J. (1990): Beyond formal procedures: The interpersonal context of procedural justice. In J.S. Carroll (Hrsg.): Applied social psychology and organizational settings. Hillsdale, NJ: Lawrence Erlbaum Associates: 77-98.

Vanderwerf, P.A. \& Brush, C.G. (1989): Achieving empirical progress in an undefined field. Entrepreneurship: Theory and Practice, 14: 45-58.

Venture Capital Report (1996): The venture capital report: Guide to venture capital in the UK and Europe. Bath: Bath Press.

Ward P.T., Duray R., Leong G.K. \& Sum C.-C. (1995): Business environment, operations strategy, and performance: An empirical study of Singapore manufacturers. Journal of Operations Management, 13: 99-115.

Weibel, A. (2007): Control, trust and trustworthiness. In: R. Lang (Hrsg.), Tagungsband Kommission Organisation 2006.

Welpe, I.M. (2004): Venture-Capital-Geber und ihre Portfoliounternehmen: Erfolgsfaktoren der Kooperation. Gabler, DUV.

West, P. G. (2007): Collective cognition: When entrepreneurial teams, not individuals, make decisions. Entrepreneurship Theory and Practice, 31(1): 77-102.

Welter, F. \& Smallbone, D. (2006): Exploring the role of trust in entrepreneurial activity. Entrepreneurship: Theory \& Practice, 30(4): 465-475.

Williamson, O.E. (1985): The economic institutions of capitalism. New York: The Free Press.

Zahra, S.A., Yavuz, R.I. \& Ucbasaran, D. (2006): How much do you trust me? The dark side of relational trust in new business creation in established companies. Entrepreneurship: Theory \& Practice, 30(4): 541559. 


\section{Die Entstehung von Vertrauen im Kontext von Unsicherheit und Informations- asymmetrie}

\section{Zusammenfassung}

Dieser Beitrag untersucht die Entstehung von Vertrauen im Kontext junger Wachstumsunternehmen und unterscheidet dabei das Vertrauen von Unternehmensgründern in die gute Absicht (Goodwill) ihres Venture-Capital-Gebers und das Vertrauen in die Kompetenz ihres Venture-Capital-Gebers. Die Ergebnisse zeigen, dass beide Arten von Vertrauen unterschiedlichen Einflussfaktoren unterliegen. So wird das Vertrauen in die guten Absichten von der Qualität der Interaktionsprozesse und das Vertrauen in die Kompetenz von den Inhalten der Interaktionen beeinflusst. Insgesamt bestätigen die Ergebnisse, dass Vertrauen in die Kompetenz und Vertrauen in die gute Absicht zwei unterschiedliche Dimensionen von Vertrauen sind, die zudem jeweils unterschiedlichen Einflussfaktoren unterliegen. Zukünftige Studien sollten daher nicht nur das Ausmaß des Vertrauens, sondern auch die Art des Vertrauens berücksichtigen.

\section{The Formation of Trust in the Context of Uncertainty and Information Asymmery}

\section{Summary}

This paper examines the antecedents of goodwill and competence trust in the venture capital context from the entrepreneur's perspective. Based on recent survey data from 272 venture capital-financed portfolio companies in German-speaking Europe, this study shows that different antecedents predict goodwill and competence trust. Goodwill trust is influenced by deal fairness and openness of the due diligence, whereas competence trust is influenced by needs assessment and deal fairness. In sum, the results confirm that goodwill and competence trust are distinct dimensions of trust that have a distinct pattern of association to antecedent variables. Future research should therefore consider not only the level but also the form of trust. 